\title{
Introduction to renormalization
}

\author{
Yu.Holovatch ${ }^{1,2,3}$ \\ ${ }^{1}$ Institute for Condensed Matter Physics of the National Academy of Sciences of Ukraine, \\ 1 Svientsitskii Str., 79011 Lviv, Ukraine \\ 2 Institute für Theoretische Physik, Johannes Kepler Universität Linz, A-4040, Linz, Austria \\ 3 Ivan Franko National University of Lviv, 79005 Lviv, Ukraine
}

Received February 13, 2006, in final form April 17, 2006

In these lectures I discuss peculiarities of the critical behaviour of "non-ideal" systems as it is explained by the renormalization group approach. Examples considered here include account of the single-ion anisotropy, structural disorder, frustrations. I introduce main ideas of renormalization and show how it serves the explanation of typical features of criticality in the above systems: softening of the phase transition, changes in the universality class, complicated effective critical behaviour.

Key words: critical behaviour, renormalization, field-theoretical renormalization group

PACS: $05.50 .+q, 05.70 . J k, 64.60 . A k$

\section{Contents}

2 Criticality and scaling

3 Non-ideal spin Hamiltonians: single ion anisotropy, structural disorder, frustrations 240

3.1 An ideal system: $m$-vector model . . . . . . . . . . . . . . . . . . . . . . . . . . . 240

3.2 Single-ion anisotropy . . . . . . . . . . . . . . . . . . . . . . . . . 240

3.3 Structural disorder . . . . . . . . . . . . . . . . . . . . . . . . . . . . . 241

3.3.1 Random-site dilution . . . . . . . . . . . . . . . . . . . . . . . . . . 241

3.3 .2 Random anisotropy . . . . . . . . . . . . . . . . . . . . . . . . . . 242

3.4 Frustrations . . . . . . . . . . . . . . . . . . . . . . . . . 242

4 Renormalization $\quad 243$

4.1 RG transformation . . . . . . . . . . . . . . . . . . . . . . . 243

4.2 Fixed points and RG flows . . . . . . . . . . . . . . . . . . . 245

5 From the spin Hamiltonians to the effective ones $\quad 247$

$5.1 m$-vector model . . . . . . . . . . . . . . . . . . . . . . . . 247

5.2 Cubic model . . . . . . . . . . . . . . . . . . . . . . . . . 249

5.3 Weakly diluted quenched $m$-vector model . . . . . . . . . . . . . . . . . . . 249

5.4 Random anisotropy model . . . . . . . . . . . . . . . . . . . . . . . 251

5.5 Stacked triangular antiferromagnet . . . . . . . . . . . . . . . . . . . 252

6 RG explanation of criticality in non-ideal systems 252

$6.1 m$-vector magnet . . . . . . . . . . . . . . . . . . . . . . 253

6.2 Cubic model . . . . . . . . . . . . . . . . . . . . . . . . . . 254

6.3 Random-site dilution . . . . . . . . . . . . . . . . . . . . . . . . . . 255

6.4 Random anisotropy . . . . . . . . . . . . . . . . . . . . . . . 256

6.5 Stacked triangular antiferromagnet . . . . . . . . . . . . . . . . . . 257

$\begin{array}{llr}7 & \text { Conclusions and outlook } & 258\end{array}$ 


\section{Introduction}

There exist many ways one can choose to approach the subject of these lectures. Taken that the presentation is limited in time (spent by the students and lecturers at the Caribbean seashore during the Mochima school in theoretical physics) and in space (given by the Editors of this volume) this choice becomes a difficult one. The way of presentation I decided to follow was chosen for several reasons. Extremely high theoretical level of certain modern renormalization group (RG) studies of criticality in different systems (i.e. the "language" of these studies) sometimes does not allow uninitiated reader to follow the derivations and even to understand the problem statement and/or physical consequences of the results. Therefore the goal would be to provide a minimal vocabulary, explaining main notions as simple as possible. On the other hand, it is tempting to use such a simple vocabulary to make a short review of state-of-the art RG studies in a certain domain. RG explanation of criticality in "non-ideal" 3d systems might be a good candidate for such a domain: it is a subject of ongoing activity where important results have recently been obtained and still a lot is to be done.

The following account will serve this purpose: after mentioning several examples of criticality and scaling in condensed matter physics (section 2) and introducing model Hamiltonians of the "non-ideal" systems we shall be interested in (section 3) I shall give the main ideas and notions of the renormalization taking as an example a simple 1d Ising model (section 4). Once the reader is acquainted with the RG transformation, its flow and fixed points, stability, universality and scaling, I shall pass to the "non-ideal" systems showing how to obtain their effective Hamiltonians and to the reviewing of recent results in this domain (sections 5 and 6). Some conclusions and outlook are given in section 7 .

\section{Criticality and scaling}

It is generally recognized that the term "critical point" was introduced in 1869 by Thomas Andrews who studied a special point for carbon dioxide at about $31^{\circ} \mathrm{C}$ and 73 atmospheres pressure where the properties of liquid and of gas become indistinguishable [1]. Approaching the critical point $T_{\mathrm{c}}$ from below, the liquid-gas density difference obeys a power law scaling governed by the critical exponent $\beta$ :

$$
\rho_{\mathrm{L}}-\rho_{\mathrm{G}} \propto\left(T_{\mathrm{c}}-T\right)^{\beta}, \quad T \rightarrow T_{\mathrm{c}}^{-},
$$

as shown in the figure 1a. Similar power laws were found for some other physical quantities,

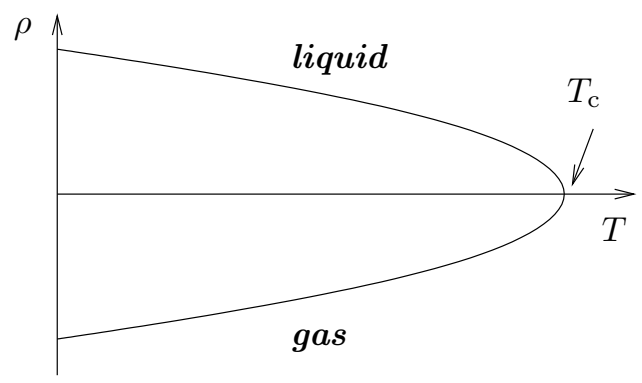

(a)

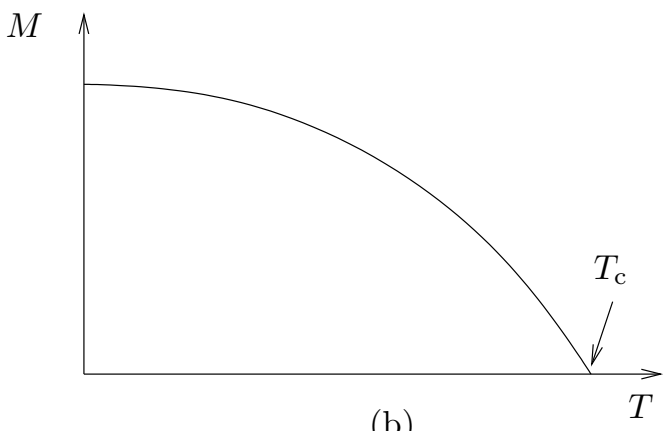

(b)

Figure 1. Order parameters for the critical point of fluids (a) and Curie point of magnets (b).

describing their approach to zero or singularity at $T_{\mathrm{c}}$. Less known is the fact, that the mean field values of critical exponents (which are integers or their simple relations, e.g. $\beta=1 / 2$ for 
equation (2.1)) were questioned already over a century ago $[2]^{1}$. Therefore much time passed before there appeared the theory that both explained scaling behaviour (2.1) found in condensed matter physics, and, on a larger scale, not only in physics, as well as offered quantitatively accurate predictions for the exponents. As we now know this theory is based on the renormalization group formalism [3] and has its origin in quantum field problems [4].

Next experimental observations of critical behaviour arrived in 1895, when Pierre Curie showed that the ferromagnet iron also displayed a special point. This point is the highest temperature at which iron can be permanently magnetized at zero external magnetic field which is now called the Curie point. Curie himself noticed the parallelism between density-temperature curve at constant pressure for carbon dioxide and magnetization-temperature curves at constant magnetic field for iron. Behaviour of the order parameter, spontaneous magnetization $M$, in the vicinity of Curie point (we denote it $T_{\mathrm{c}}$ as well) is governed by the familiar scaling law, cf. equation (2.1):

$$
M \propto\left(T_{\mathrm{c}}-T\right)^{\beta}, \quad T \rightarrow T_{\mathrm{c}}^{-} .
$$

It appears that different magnetic systems possess different sets of critical exponents but there exist wide classes of magnets characterized by the same values of exponents. For example, uniaxial magnets (like $\mathrm{FeF}_{2}, \mathrm{MnF}_{2}, \mathrm{~K}_{2} \mathrm{CoF}_{4}, \mathrm{Rb}_{2} \mathrm{CoF}_{4}$ ) possess the same critical exponents. Moreover, they coincide with those of simple fluids $\left(\mathrm{Xe}, \mathrm{SF}_{6}, \mathrm{CO}_{2} \ldots\right)$ and some other systems. In such a case it is said that these systems belong to the same universality class. The reasons for this as well as the main characteristics of a system which define the universality classes will be cleared up in the subsequent sections.

As far as the second derivatives of Gibbs free energy (isothermal magnetic susceptibility for magnets and isothermal compressibility for fluids) diverge at $T_{\mathrm{c}}$ such phase transitions are referred to as the second order phase transitions. This classification is due to Paul Ehrenfest. The order parameter does not possess a discontinuity at $T_{\mathrm{c}}$ : it is an example of a continuous phase transition. Phenomena which occur in the vicinity of the 2 nd order phase transition point are called critical phenomena. Changes of system structure at $T_{\mathrm{c}}$ are reflected in the behaviour of the pair correlation function $G_{2}(r)$ (density-density function for fluids and magnetization-magnetization one for magnets) and described by two universal exponents $\eta$ and $\nu$. The exponential decay of $G_{2}(r)$ with distance at $T>T_{\mathrm{c}}$ transforms to the power law one:

$$
G_{2}(r) \sim \mathrm{e}^{-r / \xi}, \quad T>T_{\mathrm{c}} ; \quad G_{2}(r) \sim \frac{1}{r^{d-2+\eta}}, \quad T=T_{\mathrm{c}},
$$

where $\xi$ is a correlation length which diverges at $T_{\mathrm{c}}: \xi \sim\left|T-T_{\mathrm{c}}\right|^{-\nu}, T \rightarrow T_{\mathrm{c}}$.

The XX century witnessed numerous experiments performed after pioneering works of Thomas Andrews and Pierre Curie where it was shown that criticality and scaling accompany not only the second order thermodynamic phase transitions. They are found in quantum phase transitions, percolation, non-equilibrium dissipative phase transitions. Properties of long flexible polymer chains in good solvents are described in terms of critical phenomena as well. This list can be continued. However already the above mentioned field of phenomena is very wide, especially if one takes into account that any of them occurs in objects differing by their microscopic nature. Thermodynamic 2nd order phase transitions occur in magnets, ferroelectrics, quantum liquids; percolation phenomena occurs in resistor networks, random magnets, gas filters. Nevertheless there are at least two essential features common for the above mentioned phenomena. These are: the singular character of change of properties in certain critical points and universal behaviour in the vicinity of these points. As it became clear now the reason for both is anomalous growth of fluctuations and their correlation at very high distances in the vicinity of critical points. Correlation range becomes the only characteristic scale of the system and this causes the insensitivity of its behaviour to the so-called microscopic parameters. In particular this results in the scale invariance of the system where critical phenomena occur.

Since the pioneering work of Ising [5], theoretical description of many-particle systems is often based on the so-called classical spin models. They appeared to be of primary importance to reveal

\footnotetext{
${ }^{1}$ I thank Reinhard Folk for pointing me the work of Jules-Émile Verschaffelt.
} 
the main features of criticality. In the next section 3 we shall discuss several models currently used in describing the critical phenomena, the analysis of which will be a subject of subsequent sections.

\section{Non-ideal spin Hamiltonians: single ion anisotropy, structural disorder, frustrations}

In classical spin models, each particle is imitated as a vector located on a lattice site and "interacting" with other vectors. The interaction is chosen to mimic the interparticle one whereas the dimensionality of the vector is equal to the number of internal degrees of freedom of the particle. Below we start with what we call an ideal system, which is a $m$-vector model and show how to include different types of non-idealities within a spin model formalism.

\subsection{An ideal system: $m$-vector model}

The model describes a system of $m$-dimensional classical vectors ("spins") located in the sites of $d$-dimensional hypercubic lattice. The Hamiltonian of the $m$-vector model in the absence of an external magnetic field reads:

$$
\mathcal{H}=-\frac{1}{2} \sum_{\mathbf{R}, \mathbf{R}^{\prime}} J\left(\left|\mathbf{R}-\mathbf{R}^{\prime}\right|\right) \vec{S}_{\mathbf{R}} \vec{S}_{\mathbf{R}^{\prime}},
$$

where $J\left(\left|\mathbf{R}-\mathbf{R}^{\prime}\right|\right)$ is an interaction between spins $\vec{S}_{\mathbf{R}}$ and $\vec{S}_{\mathbf{R}^{\prime}}$ located in sites $\mathbf{R}$ and $\mathbf{R}^{\prime}$ (we shall consider it to be the short-range one and of ferromagnetic origin: $J(R)>0)$ and $\vec{S}_{\mathbf{R}} \vec{S}_{\mathbf{R}^{\prime}}$ means a scalar product. Such a model appears as a natural generalization of the Ising model for the case of $m$-component spin [6] and serves as a basic model in describing phase transitions in systems with multicomponent order parameter. Below, we shall be mainly interested in the $3 \mathrm{~d}$ systems. However, for the sake of completeness let us recall that ferromagnetic ordering does not occur in this model at $d=1$ [6], it does not occur for $d=2$ and $m>1$ either [7]. Whereas the celebrated Onsager solution [8] of the $2 \mathrm{~d}$ Ising model $(d=2, m=1)$ brings about the second order phase transition with non-trivial values of the critical exponents: $\beta=1 / 8, \nu=1, \eta=1 / 4, \gamma=7 / 4$, $\alpha=0$ (exponents $\gamma$ and $\alpha$ govern power law scaling of the magnetic susceptibility and specific heat, the latter being logarithmically divergent for the $2 \mathrm{~d}$ Ising model). At $d=3$, ferromagnetic ordering occurs at any $m$ (see table 1 for typical values of exponents), and $d=4$ is the upper critical dimension of the problem: exponents attain their mean field values for $d \geqslant 4$.

Table 1. The standard values of the critical exponents of the $3 \mathrm{~d} m$-vector model (obtained in [9] from the high-order renormalization group expansions).

\begin{tabular}{|c|c|c|c|c|c|}
\hline$m$ & $\gamma$ & $\nu$ & $\eta$ & $\beta$ & $\alpha$ \\
\hline 1 & $1.2396(13)$ & $0.6304(13)$ & $0.0335(250)$ & $0.3258(14)$ & $0.109(4)$ \\
\hline 2 & $1.3169(20)$ & $0.6703(15)$ & $0.0354(250)$ & $0.3470(16)$ & $-0.011(4)$ \\
\hline 3 & $1.3895(50)$ & $0.7073(35)$ & $0.0355(250)$ & $0.3662(25)$ & $-0.122(10)$ \\
\hline
\end{tabular}

We refer to the above model as to the ideal one: it describes the lattice system without any defects of structure or complexities of interaction which are often encountered in real systems. Let us show how those can be considered within the same formalism.

\subsection{Single-ion anisotropy}

Real substances often are anisotropic. For instance, in cubic crystals one expects the spin interaction to react on the lattice structure (crystalline anisotropy) suggesting additional terms in the Hamiltonian, invariant under the cubic group [10]. Such a single-ion anisotropy breaks the 
rotational symmetry of the $m$-vector magnet (3.1) and the Hamiltonian reads:

$$
\mathcal{H}=-\frac{1}{2} \sum_{\mathbf{R}, \mathbf{R}^{\prime}} J\left(\left|\mathbf{R}-\mathbf{R}^{\prime}\right|\right) \vec{S}_{\mathbf{R}} \vec{S}_{\mathbf{R}^{\prime}}+V \sum_{\mathbf{R}} \sum_{i=1}^{m}\left(S_{\mathbf{R}}^{i}\right)^{4}
$$

where $V$ defines anisotropy strength and makes the order parameter to point either along the edges $(V>0)$ or along diagonals $(V<0)$ of a $m$-dimensional hypercube. Therefore a model with the spin Hamiltonian (3.2) is often called a cubic model.

An interesting phenomenon is observed in 3d cubic magnets: for low values of $m$ their critical exponents coincide with those of the "ideal" $m$-vector model (one speaks about isotropization of critical fluctuations), whereas for large $m$ they belong to the new universality class. The value $m_{\mathrm{c}}^{\mathrm{cub}}$ at which one regime is changed to the other one is called the marginal dimension. Moreover, transition to the low-temperature phase may also occur via the first-order scenario.

\subsection{Structural disorder}

To treat structural disorder within the lattice model one usually introduces random variables into the Hamiltonian (3.1) and couples them to spin degrees of freedom. The new model can either mimic a quenched system (new variables are randomly distributed and fixed in a certain configuration) or an annealed, equilibrium one [11]. Here, we shall be interested in changes of critical behaviour caused by quenched disorder. Furthermore, we shall consider two different examples, showing how to introduce disorder via dilution and random anisotropy ${ }^{2}$.

\subsubsection{Random-site dilution}

To describe dilution, one may introduce the random-site $m$-vector model, considering situation, when spins $\vec{S}_{\mathbf{R}}$ in (3.1) occupy only a part of the lattice sites, $N_{1}$, the rest $N-N_{1}$ sites being empty (or occupied by non-magnetic atoms). Magnetic and non-magnetic sites are randomly distributed and fixed in a certain configuration. The model Hamiltonian reads:

$$
\mathcal{H}=-\frac{1}{2} \sum_{\mathbf{R}, \mathbf{R}^{\prime}} J\left(\left|\mathbf{R}-\mathbf{R}^{\prime}\right|\right) c_{\mathbf{R}} c_{\mathbf{R}^{\prime}} \vec{S}_{\mathbf{R}} \vec{S}_{\mathbf{R}^{\prime}}
$$

where $c_{\mathbf{R}}$ are the occupation numbers:

$$
c_{\mathbf{R}}= \begin{cases}1, & \text { site } \mathbf{R} \text { is occupied } \\ 0, & \text { site } \mathbf{R} \text { is empty }\end{cases}
$$

Here we do not touch upon the phenomena occuring near the percolation threshold $c_{\text {perc }}$ and consider the so-called weak dilution, $c=N_{1} / N \gg c_{\text {perc }}$. To complete the model one should choose a certain distribution function for the occupation numbers $c_{\mathbf{R}}$. Let us consider the case when the site is occupied with the probability $c$ and is empty with the probability $(1-c)$ and this probability does not depend on the occupation numbers on the neighbouring sites. Such a situation corresponds to the following occupation probability $\mathcal{P}\left(\left\{c_{\mathbf{R}}\right\}\right)$ :

$$
\mathcal{P}\left(\left\{c_{\mathbf{R}}\right\}\right)=\prod_{\mathbf{R}} p\left(c_{\mathbf{R}}\right), \quad p\left(c_{\mathbf{R}}\right)=c \delta\left(c_{\mathbf{R}}-1\right)+(1-c) \delta\left(c_{\mathbf{R}}\right)
$$

For different $m$, the above model describes magnetic phase transitions in crystalline alloys of uniaxial magnets and their non-magnetic isomorphs $\mathrm{Fe}_{\mathrm{x}} \mathrm{Zn}_{1-\mathrm{x}} \mathrm{F}_{2}, \mathrm{Mn}_{\mathrm{x}} \mathrm{Zn}_{1-\mathrm{x}} \mathrm{F}_{2}$ [13], diluted Heisenberglike magnets $[14,15]$, superfluid phase transition in $\mathrm{He}^{4}$ in porous medium [16]. Similar to the cubic anisotropy systems, there exists a marginal dimensionality $m_{\mathrm{c}}^{\text {dil }}$ separating two different scenarios:

\footnotetext{
${ }^{2}$ Another option would be to consider a random field disorder [12]. However, we do not introduce it since an appropriate model differs from those considered here by its upper critical dimension and this will make our account even broader. The same concerns strong dilution at the percolation threshold.
} 
for $m<m_{\mathrm{c}}^{\text {dil }}$ dilution causes a change in the universality class, whereas the asymptotic critical exponents of $m>m_{\mathrm{c}}^{\mathrm{dil}}$-component systems remain unchanged under dilution. The so-called Harris criterion allows us to determine this marginal dimension from the pure system heat capacity behaviour [17]. It states that critical exponents are not altered by dilution, if the heat capacity of the pure system does not diverge $(\alpha<0)$. From Table 1 one concludes that in three dimensions it is the Ising model which changes the universality class.

\subsubsection{Random anisotropy}

Another way of introducing randomness to the model (3.1) is to consider the case, when each spin is subjected to a local anisotropy of random orientation. The resulting Hamiltonian reads [18]:

$$
\mathcal{H}=-\frac{1}{2} \sum_{\mathbf{R}, \mathbf{R}^{\prime}} J\left(\left|\mathbf{R}-\mathbf{R}^{\prime}\right|\right) \vec{S}_{\mathbf{R}} \vec{S}_{\mathbf{R}^{\prime}}-D \sum_{\mathbf{R}}\left(\hat{x}_{\mathbf{R}} \vec{S}_{\mathbf{R}}\right)^{2}
$$

Here, $D>0$ is the strength of the anisotropy and $\hat{x}_{\mathbf{R}}$ is a random unit vector pointing in the direction of the local anisotropy axis. The random anisotropy model (3.6) is relevant to the description of a wide class of disordered magnets. It was first introduced to describe magnetic properties of amorphous alloys of rare-earth compounds with aspherical electron distributions and transition metals [18]. Today the majority of the amorphous alloys containing rare-earth elements are recognized to be random anisotropy magnets [19].

As in the random-site case, the model should be completed by choosing a certain distribution $p\left(\hat{x}_{\mathbf{R}}\right)$ for the random variables $\hat{x}_{\mathbf{R}}$. Most often, two different distributions are considered [21]. The first is an isotropic one, where the random vector $\hat{x}_{\mathbf{R}}$ points with equal probability in any direction in the $m$-dimensional hyperspace:

$$
p\left(\hat{x}_{\mathbf{R}}\right) \equiv\left(\int \mathrm{d}^{m} \hat{x}_{\mathbf{R}}\right)^{-1}=\frac{\Gamma(m / 2)}{2 \pi^{m / 2}} .
$$

Here $\Gamma(x)$ is Euler gamma-function, and the right-hand side presents the volume of the $m$ dimensional hypersphere of unit radius. This distribution mimics an amorphous system without any preferred direction. The second distribution restricts the vector $\hat{x}_{\mathbf{R}}$ to point with equal probability along one of the $2 m$ directions of the axes $\hat{k}_{i}$ of a hypercubic lattice:

$$
p\left(\hat{x}_{\mathbf{R}}\right)=\frac{1}{2 m} \sum_{i=1}^{m}\left[\delta^{(m)}\left(\hat{x}_{\mathbf{R}}-\hat{k}_{i}\right)+\delta^{(m)}\left(\hat{x}_{\mathbf{R}}+\hat{k}_{i}\right)\right]
$$

where $\delta(y)$ are Dirac $\delta$-functions. This distribution (sometimes called a cubic one) corresponds to a situation when an amorphous magnet still remembers the initial cubic lattice structure.

It is generally believed that ferromagnetism does not exist for the $3 \mathrm{~d}$ model (3.6) with the isotropic random axis distribution (3.7) [19]. However, anisotropic distribution (3.8) leads to a magnetically ordered low temperature phase and the transition belongs to the random site Ising model universality class [20].

\subsection{Frustrations}

An archetype of a model describing the effect of frustrations is the model of stacked triangular antiferromagnet [22]. In this model, the antiferromagnetically interacting spins are placed on the sites of $2 \mathrm{~d}$ triangular lattices stacked in register along the orthogonal direction. The sign of the interlayer interaction is unimportant, because there are no frustrations in orthogonal direction. The Hamiltonian reads:

$$
\mathcal{H}=-\frac{1}{2} \sum_{\left\langle\mathbf{R}, \mathbf{R}^{\prime}\right\rangle} J \vec{S}_{\mathbf{R}} \vec{S}_{\mathbf{R}^{\prime}}, \quad J= \begin{cases}J_{1}<0, & \text { inside a plane, } \\ J_{2}, & \text { between planes }\end{cases}
$$


Sum in (3.9) runs over the nearest neighbours of the above described lattice. Systems which are characterized by the Hamiltonian (3.9) exhibit noncollinear spin ordering. An example is given by the famous $120^{\circ}$ structure: each spin in a layer forms $120^{\circ}$ angles with the neighbouring spins. Although model (3.9) is formulated for general $m$, of most interest are values $m=2$ and $m=$ 3. Namely for these values of $m$ the model has experimental realizations [14,23] and describes noncollinear ordering of stacked triangular antiferromagnets as $\mathrm{VCl}_{2}, \mathrm{VBr}_{2}, \mathrm{CsMnBr}_{3}$, and helical magnets as $\mathrm{Ho}, \mathrm{Dy}, \beta-\mathrm{MnO}_{2}$. In the latter substances, frustration is induced by the competition of ferromagnetic nearest-neighbour and antiferromagnetic next-nearest-neighbour interactions, which acts only along one lattice axis: $J_{1}>0$ and $J_{2}<0$, correspondingly. When the ratio $J_{1} / J_{2}$ exceeds a critical value, in the low-temperature phase the spins align ferromagnetically in a plane and form a spiral along the orthogonal axis. Moreover, at $m=3$ Hamiltonian (3.9) also describes A/B transition in $\mathrm{He}^{3}$.

Numerous experimental and MC studies performed so far have not lead to the definitive conclusion about the order of transition into non-collinear state. There is no unique answer from the theoretical viewpoint either [14,23].

\section{Renormalization}

In order to explain the main ideas of the renormalization group (RG) theory and to show how this method works in practice we shall study the critical behaviour of the 1d Ising model by means of the RG approach. The explanations given below are due to Michael Fisher [24], who compared this study with the Bohr-Sommerfeld picture in quantum mechanics. Passing from the classical mechanics to the full account of quantum mechanics, Bohr-Sommerfeld's picture represents only a crude approximation. Nevertheless, it introduces some important ideas, just like RG study of the 1d Ising model enables one to introduce ideas of renormalization and scaling in the critical region.

\subsection{RG transformation}

We start from the Hamiltonian of the 1d Ising model in the presence of an external magnetic field $H$, which being divided by $k_{\mathrm{B}} T$ is written in the following form:

$$
-\mathcal{H}_{\mathrm{eff}}=K \sum_{j=1}^{N} S_{j} S_{j+1}+h \sum_{j=1}^{N} S_{j}+C \sum_{j=1}^{N} 1,
$$

where $K=J /\left(k_{\mathrm{B}} T\right), h=H /\left(k_{\mathrm{B}} T\right)$, and the last term $C \sum_{j=1}^{N} 1=N C$ is added for the convenience of forthcoming calculations. We call this temperature-dependent Hamiltonian an effective one, $\mathcal{H}_{\text {eff }}$. Note, however, that contrary to the effective Hamiltonians considered in the forthcoming section 5 , it contains full information about the spin Hamiltonian. Given $\{K, h, C\}$ one completely specifies $\mathcal{H}_{\text {eff }}$ : thus it can be regarded as a point in a space of 3 parameters $\{K, h, C\}$. With change of $\{K, h, C\}$, this point moves.

One of the first approaches to the $\mathrm{RG}$ is to regard it as a specific way of calculating the partition function $Z_{N}\{\mathcal{H}\}$ :

$$
Z_{N}\left\{\mathcal{H}_{\text {eff }}\right\}=\frac{1}{2^{N}} \sum_{S_{1}= \pm 1} \cdots \sum_{S_{N}= \pm 1} \mathrm{e}^{-\mathcal{H}_{\text {eff }}}
$$

where we have normalized the expression for the partition function by the partition function of the ideal model $\left(Z^{\text {ideal }}=2^{N}\right)$ to obtain $Z_{N}\left\{\mathcal{H}_{\text {eff }}\right\} \rightarrow_{T \rightarrow \infty}$ 1. The RG method of calculating $Z_{N}$ is to step-by-step perform the summation in (4.2) in such a way as to try as much as possible to preserve the system as it used to be prior the summation.

Let us do this by means of decimation procedure: performing summation over every second spin variable (see figure 2). To show the changes which are introduced by summation over certain spin variable $S_{0}$ let us represent the total Boltzmann weight in the factorized form:

$$
\mathrm{e}^{-\mathcal{H}_{\text {eff }}}=\cdots \mathrm{e}^{K S_{-} S_{0}+\frac{1}{2} h\left(S_{-}+S_{0}\right)+C} \mathrm{e}^{K S_{0} S_{+}+\frac{1}{2} h\left(S_{0}+S_{+}\right)+C} \cdots=\cdots P\left(S_{-}, S_{0}\right) P\left(S_{0}, S_{+}\right) \cdots
$$




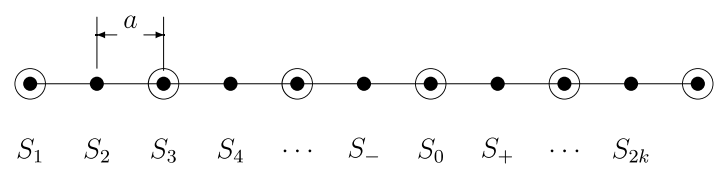

partial trace

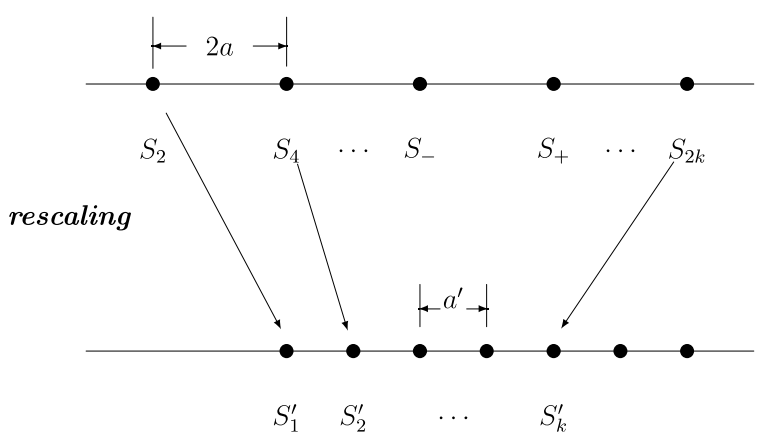

Figure 2. Decimation procedure followed by the spatial rescaling. Summation over every second spin variable is performed and then all lengths are rescaled in such a way that the new lattice spacing $a^{\prime}$ is equal to the old one $a$. Now any distance $R$ in the original lattice becomes $R^{\prime}=1 / 2 R$ in the new one measured in the units of the lattice spacing.

Summation over $S_{0}$ which enters only $P\left(S_{-}, S_{0}\right)$ and $P\left(S_{0}, S_{+}\right)$will lead to a new function $P^{\prime}\left(S_{-}, S_{+}\right)$, defined by:

$$
P^{\prime}\left(S_{-}, S_{+}\right)=\frac{1}{2} \sum_{S_{0}= \pm 1} P\left(S_{-}, S_{0}\right) P\left(S_{0}, S_{+}\right) .
$$

Factor $1 / 2$ is included in (4.4) because with each spin eliminated one must remove factor $\frac{1}{2}$ from the normalizing factor in (4.2). Now the RG idea is to express the new factor $P^{\prime}$ in the same form as the initial one:

$$
P^{\prime}\left(S_{-}, S_{+}\right)=\mathrm{e}^{K^{\prime} S_{-} S_{+}+\frac{1}{2} h^{\prime}\left(S_{-}+S_{+}\right)+C^{\prime}} .
$$

The new parameters define the renormalized Hamiltonian:

$$
\mathcal{H}_{\text {eff }}^{\prime}=\mathcal{H}_{\text {eff }}^{\prime}\left\{K^{\prime}, h^{\prime}, C^{\prime}\right\},
$$

and $\mathcal{H}_{\text {eff }}^{\prime}$ has a half of initial spins. The result can be formally written as :

$$
\mathcal{H}_{\text {eff }}^{\prime}=\mathcal{R}_{b}\left[\mathcal{H}_{\text {eff }}\right],
$$

with a spatial rescaling factor $b$ (in our case $b=2$, see figure 2). The resulting number of spins $N^{\prime}$ is connected with the initial one by $N^{\prime}=N / b$, and in the case of $d$-dimensional system this is generalized to:

$$
N^{\prime}=N / b^{d} \text {. }
$$

Performing summation (4.4) and expressing the result in the renormalized form (4.5) one can get expressions for the renormalized variables $K^{\prime}, h^{\prime}, C^{\prime}$ (it is proposed to the interested reader to do this).

Now the renormalized model is characterized by the Hamiltonian $\mathcal{H}_{\mathrm{eff}}^{\prime}\left(K^{\prime}, h^{\prime}, C^{\prime}\right)$ having the form similar to the initial one, though the lattice constant after taking the sum over every second spin is equal to $2 a$ (see figure 2). In order to have the renormalized model looking as the initial one we rescale all lengths in such a way that the new lattice spacing $a^{\prime}$ is equal to the old one. Now any distance $R$ in the original lattice becomes $R^{\prime}=1 / 2 R$ in the new one measured in the units of the lattice spacing. For the arbitrary $b$ we have the following mapping:

$$
R \Rightarrow R^{\prime}=R / b \text {. }
$$


Now let us have a look at the behaviour of the spin-spin correlation function $\left\langle S_{0} S_{R}\right\rangle$. First let us renumber the remaining spins to have the labels arranged in the subsequent order (see figure 2): $S_{2} \Rightarrow S_{1}^{\prime}, \quad S_{4} \Rightarrow S_{2}^{\prime}, \ldots, \quad S_{2 k} \Rightarrow S_{k}^{\prime}, \ldots$ Or, taking into account (4.9), $S_{2 R^{\prime}}=S_{R^{\prime}}^{\prime}$. Since the remaining spins $S^{\prime}$ have not changed under the renormalization procedure, the renormalized correlation function is equal to the original one:

$$
\left\langle S_{0} S_{2 R^{\prime}}\right\rangle=\left\langle S_{0}^{\prime} S_{R^{\prime}}^{\prime}\right\rangle
$$

It follows that if the original correlation length is $\xi=\xi\left(\mathcal{H}_{\text {eff }}\right)$ then the renormalized correlation length is two times smaller. Or, for a general $b$ :

$$
\xi\left(\mathcal{H}_{\mathrm{eff}}\right)=b \xi\left(\mathcal{H}_{\mathrm{eff}}^{\prime}\right)
$$

The RG procedure has the effect of shrinking the correlation length. Recalling that $\xi$ becomes infinite at $T=T_{\mathrm{c}}$ one can state, that the RG procedure is driving a system away from criticality (if it was not critical). Already here it is seen that the RG transformation we are considering has a deep connection with the critical properties of a system.

\subsection{Fixed points and RG flows}

To proceed in the determination of these properties let us have a closer look at the partition function and at the free energy of the system. First, after taking a partial trace of the partition function $Z_{N}\left\{\mathcal{H}_{\text {eff }}\right\}$ we get the Boltzmann distribution in $N^{\prime}$ spin variables $S^{\prime}$ :

$$
\mathrm{e}^{-\mathcal{H}_{\text {eff }}^{\prime}\left(S^{\prime}\right)}=S p_{N^{\prime \prime}}^{S^{\prime \prime}} \mathrm{e}^{-\mathcal{H}_{\text {eff }}(S)}
$$

where $S p_{N^{\prime \prime}}^{S^{\prime \prime}}$ stands for the trace over $N^{\prime \prime}=N-N^{\prime}$ spin variables $S^{\prime \prime}$. Now taking trace over the remaining $N^{\prime}$ spin variables one gets:

$$
Z_{N^{\prime}}\left\{\mathcal{H}_{\mathrm{eff}}^{\prime}\right\}=S p_{N^{\prime}}^{S^{\prime}} \mathrm{e}^{-\mathcal{H}_{\mathrm{eff}}^{\prime}\left(S^{\prime}\right)} .
$$

Substituting into the right hand side of (4.13) its explicit form given by (4.12) one gets:

$$
Z_{N^{\prime}}\left\{\mathcal{H}_{\text {eff }}^{\prime}\right\}=S p_{N^{\prime}}^{S^{\prime}} S p_{N^{\prime \prime}}^{S^{\prime \prime}} \mathrm{e}^{-\mathcal{H}_{\text {eff }}(S)}=Z_{N}\left\{\mathcal{H}_{\mathrm{eff}}\right\}
$$

This relation can be rewritten in terms of the flow equations which describe a motion of a point describing an effective Hamiltonian:

$$
\begin{aligned}
K^{\prime} & =\mathcal{R}_{K}(K, h), \\
h^{\prime} & =\mathcal{R}_{h}(K, h), \\
C^{\prime} & =b^{d} C+\mathcal{R}_{0}(K, h),
\end{aligned}
$$

for the couplings defining $\mathcal{H}_{\text {eff }}$. As far as the temperature $T$ enters parameter $K$, the above relations imply the flow equations for $T$ as well. To study this let us consider $H=0$ and write the flow equation for the temperature as:

$$
T \Rightarrow T^{\prime}=\mathcal{R}(T),
$$

with $\mathcal{R}(T)$ being appropriate function of $T$. Suppose that $\mathcal{R}(T)$ has a form given in the figure 3 . The important feature there is that $\mathcal{R}(T)$ crosses the line $T^{\prime}=T$ at some point $T=T^{*}$. This point is called the fixed point (FP). It is clear that when $T$ is smaller or greater than $T^{*}$ the successive application of the renormalization procedure drives the system away from the fixed point. On the other hand at $T=T^{*}$ the system remains at the fixed point under the application of the RG procedure. Recalling the flow equation for the correlation length (4.11) which can be written as:

$$
\xi(T)=b \xi\left(T^{\prime}\right)
$$

one has that in the FP this reads:

$$
\xi\left(T^{*}\right)=b \xi\left(T^{*}\right) .
$$

Since $b>1$ it is possible only when 


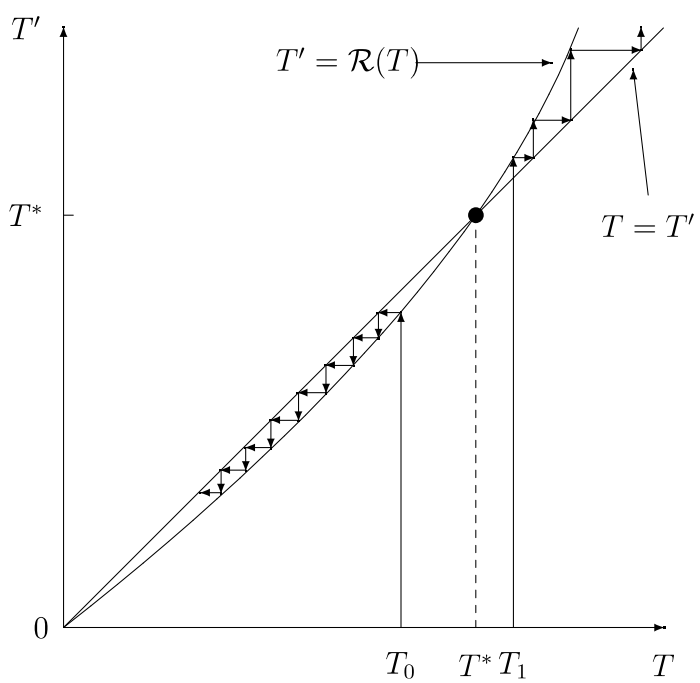

Figure 3. Temperature renormalization function $\mathcal{R}(T)$. $\mathcal{R}(T)$ crosses the line $T^{\prime}=T$ at the fixed point $T=T^{*}$. When $T$ is smaller $\left(T=T_{0}\right)$ or greater $\left(T=T_{1}\right)$ than $T^{*}$, the successive application of the renormalization procedure drives the system away from the fixed point. On the other hand at $T=T^{*}$ the system remains at the fixed point under the application of the RG procedure.

- $\xi\left(T^{*}\right)=\infty$ and

- $\xi\left(T^{*}\right)=0$.

The first case characterizes the critical point $T^{*}=T_{\mathrm{c}}$, whereas the second corresponds to the vanishing of the correlation length at zero or infinite temperatures (when the spins are frozen at a ground state or totally uncoupled). Note, that this corresponds to two FPs more $\left(T^{*}=0, T^{*}=\infty\right)$ in the figure 3.

Having identified the fixed point $T^{*}$ of the RG transformation as the critical point of the system let us study what knowledge about the critical exponents can be obtained based on the properties of the RG transformation. First let us linearize it in the vicinity of the FP introducing the variable:

$$
\tau=\frac{T-T_{\mathrm{c}}}{T_{\mathrm{c}}}=\frac{T-T^{*}}{T^{*}}
$$

and replacing the plot of $\mathcal{R}(T)$ near $T^{*}$ by its tangent at $T^{*}$.

Then after the renormalization the temperature deviation will be:

$$
\tau^{\prime} \equiv \tau^{(1)} \simeq \Lambda_{1}(b) \tau
$$

for small enough $\tau$, where $\Lambda_{1}(b)$ is the slope of the tangent. To find its dependence on $b$ let us apply the renormalization procedure twice:

$$
\tau^{\prime \prime} \equiv \tau^{(2)} \simeq \Lambda_{1}(b) \Lambda_{1}(b) \tau .
$$

Such transformation should be equivalent to transforming with a spatial rescaling factor $b^{2}$ :

$$
\tau^{(2)} \simeq \Lambda_{1}\left(b^{2}\right) \tau,
$$

or

$$
\Lambda_{1}(b) \Lambda_{1}(b)=\Lambda_{1}\left(b^{2}\right) .
$$

This leads to the conclusion that $\Lambda_{1}(b)$ should have the following form:

$$
\Lambda_{1}(b)=b^{\lambda_{1}},
$$


with $\lambda_{1}$ being constant independent of $b$. Now for the correlation length after $l$ transformations one gets:

$$
\xi(\tau)=b^{l} \xi\left(\left[\Lambda_{1}(b)\right]^{l} \tau\right)=b^{l} \xi\left(b^{l \lambda_{1}} \tau\right) .
$$

As far as (4.26) holds for any $l$ (and for small $\tau(!))$ let us choose it to satisfy:

$$
b^{l \lambda_{1}} \tau=1
$$

or

$$
b^{l}=(1 / \tau)^{1 / \lambda_{1}}
$$

Then from (4.26) one gets:

$$
\xi(\tau)=(1 / \tau)^{1 / \lambda_{1}} \xi(1)
$$

or

$$
\xi(\tau) \sim \tau^{-1 / \lambda_{1}}
$$

Comparing (4.28) with the definition of the critical exponent $\nu: \xi(\tau) \sim \tau^{-\nu}$ we get:

$$
\nu=1 / \lambda_{1} .
$$

So as we have seen, the knowledge of the linearized RG transformation enables one to determine the critical exponent!

Note, that having carried out similar analysis for the free energy per spin, $f=-N^{-1} \ln Z_{N}$, one arrives at the expression: $f(\tau)=b^{-d l} f\left(b^{\lambda_{1} l} \tau\right)$. Again, choosing $b$ from (4.27) one gets $f(\tau)=$ $\tau^{d / \lambda_{1}} f(1)=\tau^{d \nu} f(1)$. Comparing the latter with the scaling behaviour of the free energy in the vicinity of the critical point $f(\tau) \sim \tau^{2-\alpha}$ one proves the hyperscaling relation:

$$
2-\alpha=d \nu
$$

Similarly, considering the case of non-zero magnetic field one can write the recursion relations for the renormalized temperature and field. Their linearization in the vicinity of the fixed point leads to the matrix of the linear $R G$ operator with two eigenvalues, defining two different critical exponents.

To complete the RG study of the 1d Ising model it remains to perform the above described procedure explicitly and to find the values of the critical exponents (at zero-temperature FP $T=$ $H=0,[24])$. The above described transformations concisely give the main features of the RG transformation, allowing us, in particular, to define the critical exponents of the system.

\section{From the spin Hamiltonians to the effective ones}

Very often a starting point for the RG study of critical behaviour of a many-particle system is its effective Hamiltonian. Taken, that in the model description the system is identified with the spin Hamiltonian (see section 3), the effective Hamiltonian arises as a certain "metamodel". It shares global features of different spin Hamiltonians: their dimensionalities, symmetries, type of interparticle interaction and as a result, brings about common features of their critical behaviour. Below, we shall show how to obtain the effective Hamiltonians for the spin models of section 3.

\section{1. $m$-vector model}

To get an effective Hamiltonian we proceed as follows. Let us define the free energy $\mathcal{F}$ and the partition function $\mathcal{Z}$ of a spin model (3.1) as:

$$
\mathcal{F}=-\beta^{-1} \ln \mathcal{Z}, \quad \mathcal{Z}=\mathrm{Spe}^{-\beta \mathcal{H}},
$$

where $\beta=\left(k_{\mathrm{B}} T\right)^{-1}$ and $\mathrm{Sp}(\ldots)$ as usually means the sum over all possible states. In our case it corresponds to the integration over the surface of $m$-dimensional hypersphere (we take it to be of unit radius):

$$
\operatorname{Sp}(\ldots)=\prod_{\mathbf{R}} \int \mathrm{d} \vec{S}_{\mathbf{R}} \delta\left(\left|\vec{S}_{\mathbf{R}}\right|-1\right)(\ldots)
$$


Let us introduce the Fourier-transforms of the variables $\vec{S}_{\mathbf{R}}$ by:

$$
\vec{S}_{\mathbf{R}}=\frac{1}{\sqrt{N}} \sum_{\mathbf{k}} \mathrm{e}^{\mathrm{i} \mathbf{k} \mathbf{R}} \vec{S}_{\mathbf{k}}, \quad \vec{S}_{\mathbf{k}}=\frac{1}{\sqrt{N}} \sum_{\mathbf{R}} \mathrm{e}^{-\mathrm{i} \mathbf{k} \mathbf{R}} \vec{S}_{\mathbf{R}} .
$$

Here and below, when it will not be mentioned explicitly, vector $\mathbf{R}$ spans all sites of the lattice whereas $\mathbf{k}$ changes in the first Brillouin zone. Now the Hamiltonian (3.1) can be rewritten as :

$$
\mathcal{H}=-\frac{1}{2} \sum_{\mathbf{k}} \nu(k) \vec{S}_{\mathbf{k}} \vec{S}_{-\mathbf{k}}
$$

where we have introduced Fourier transform of the potential of interaction by:

$$
J(R)=\frac{1}{N} \sum_{\mathbf{k}} \mathrm{e}^{\mathrm{i} \mathbf{k} \mathbf{R}} \nu(k), \quad \nu(k)=\sum_{\mathbf{R}} \mathrm{e}^{-\mathrm{i} \mathbf{k} \mathbf{R}} J(R) .
$$

Now the partition function (5.1) reads:

$$
\mathcal{Z}=\operatorname{Spe}^{\frac{\beta}{2} \sum_{\mathbf{k}} \nu(k) \vec{S}_{\mathbf{k}} \vec{S}_{-\mathbf{k}}}
$$

In order to take the trace in (5.6) let us transform it to the expression with linear dependence on $\vec{S}_{\mathbf{k}}$. To this end, one makes use of the Stratonovich-Hubbard transformation introducing the field variable $\vec{\phi}_{\mathbf{k}}$ which is conjugated to the spin variable $\vec{S}_{\mathbf{k}}$ by the identity:

$$
\begin{aligned}
\mathrm{e}^{-\beta \mathcal{H}}= & \prod_{\vec{k}} \mathrm{e}^{\frac{\beta \nu(k)}{2} \vec{S}_{\mathbf{k}} \vec{S}_{-\mathbf{k}}}=\prod_{\vec{k}}\left(\frac{1}{2 \pi \beta \nu(k)}\right)^{m / 2} \int \mathrm{d} \vec{\phi}_{\mathbf{k}} \mathrm{e}^{\frac{-1}{2 \beta \nu(k)} \vec{\phi}_{\mathbf{k}} \vec{\phi}_{-\mathbf{k}}+\vec{S}_{\mathbf{k}} \vec{\phi}_{-\mathbf{k}}} \\
& \sim \int(\mathrm{d} \vec{\phi}) \mathrm{e}^{\sum_{\mathbf{k}}\left(\frac{-1}{2 \beta \nu(k)}\left|\vec{\phi}_{\mathbf{k}}\right|^{2}+\vec{S}_{\mathbf{k}} \vec{\phi}_{-\mathbf{k}}\right)} .
\end{aligned}
$$

As far as the Stratonovich-Hubbard transformation was performed for each function $\vec{S}_{\mathbf{k}}$ we arrived at the functional integral $\int(\mathrm{d} \vec{\phi})=\prod_{\mathbf{R}} \prod_{i=1}^{m} \int_{-\infty}^{\infty} \mathrm{d} \phi_{\mathbf{R}}^{(i)}$ over the field variables

$$
\vec{\phi}_{\mathbf{R}}=\frac{1}{\sqrt{N}} \sum_{\mathbf{k}} \mathrm{e}^{\mathrm{i} \mathbf{k} \mathbf{R}} \vec{\phi}_{\mathbf{k}}, \quad \vec{\phi}_{\mathbf{k}}=\frac{1}{\sqrt{N}} \sum_{\mathbf{R}} \mathrm{e}^{-\mathrm{i} \mathbf{k} \mathbf{R}} \vec{\phi}_{\mathbf{R}} .
$$

From now on we omit the coefficients in front of the functional integral.

We have reached our goal: now the trace in the expression for the partition function concerns only the last term of the exponents under integration in (5.6) which is a linear function of $\vec{S}$ :

$$
\mathcal{Z}=\operatorname{Spe}^{\frac{\beta}{2} \sum_{\mathbf{k}} \nu(k) \vec{S}_{\mathbf{k}} \vec{S}_{-\mathbf{k}}} \sim \int(\mathrm{d} \vec{\phi}) \mathrm{e}^{\frac{-1}{2} \sum_{\mathbf{k}} \frac{1}{\beta \nu(k)}\left|\vec{\phi}_{\mathbf{k}}\right|^{2}} \operatorname{Sp}^{\sum_{\mathbf{R}} \vec{S}_{\mathbf{R}} \vec{\phi}_{\mathbf{R}}}
$$

The last step is to take integral over $\vec{S}_{\mathbf{R}}$ (recall that the trace is defined by equation (5.2)). This can be achieved by passing from the $m$-dimensional Cartesian coordinates $S^{1}, S^{2}, \ldots, S^{m}$ to the $m$-dimensional polar ones $S, \theta_{1}, \ldots, \theta_{m-1}$. Performing this integration and representing the result as a series in $\vec{\phi}$ one gets for the partition function:

$$
\mathcal{Z} \sim \int(\mathrm{d} \vec{\phi}) \mathrm{e}^{\frac{-1}{2} \sum_{\mathbf{k}}\left(\frac{1}{\beta \nu(k)}-u_{2}\right)\left|\vec{\phi}_{\mathbf{k}}\right|^{2}-\sum_{\mathbf{R}} \sum_{l=2}^{\infty} \frac{u_{2 l} l}{(2 l) !}\left|\vec{\phi}_{\mathbf{R}}\right|^{2 l}}
$$

where the coefficients $u_{2 l}$ readily follow: $u_{2}=-1 / m, u_{4}=6 / m^{2}(m+2), \ldots$ Expression $(5.10)$ gives the functional representation of the partition function of the $m$-vector model. So far we have not gained a lot by the above described transformations: the difficulty of taking Sp of the initial expression (5.4) now is transformed into the difficulty of calculating a functional integral (5.10). However, in order to study critical behaviour of the model (3.1), expression (5.10) can be further approximated. It appears that all of $\phi$ higher than the fourth powers do not effect the asymptotic 
critical behaviour at $d=3$ : they do not change the value of the fixed point of $\mathrm{RG}$ transformation (cf. section 4) and are irrelevant in the RG sense [3,4]. Therefore one can be restricted to the $\phi^{4}$ model. Being interested in the long-range correlations arising in the system in the vicinity of a critical point, one substitutes the Fourier image of the interaction potential by its expansion for small wave vector values $k: \nu(k) \simeq \nu(0)-1 / 2\left|\nu^{\prime \prime}(0)\right| k^{2}$ and writes the contribution $\sum_{\mathbf{k}} k^{2} \vec{\phi}_{\mathbf{k}} \vec{\phi}_{-\mathbf{k}}$ in the form $\sum_{\mathbf{R}}\left(\nabla \vec{\phi}_{\mathbf{R}}\right)^{2}$ re-scaling variables $\phi$ to get the gradient term without any coefficient. One further passes to the continuous limit $\sum_{\mathbf{R}} \rightarrow \int \mathrm{d} \mathbf{R}$ and gets for the free energy:

$$
\mathcal{F} \sim \ln \int(\mathrm{d} \vec{\phi}) \mathrm{e}^{-\mathcal{H}_{\text {eff }}}
$$

with the effective Hamiltonian:

$$
\mathcal{H}_{\text {eff }}=\int \mathrm{d}^{d} R\left\{\frac{1}{2}\left((\nabla \phi)^{2}+\mu_{0}^{2} \phi^{2}\right)+\frac{u_{0}}{4 !} \phi^{4}\right\},
$$

where $\phi \equiv \vec{\phi}_{\mathbf{R}}, \phi^{2} \equiv\left|\vec{\phi}_{\mathbf{R}}\right|^{2}$, variables $\mu_{0}$ and $u_{0}$ are called bare mass and coupling. From the above derivation we know that $u_{0}$ is positive $\left(u_{0} \sim u_{4}\right.$ from equation (5.10)), which ensures the existence of the integral (5.11). Effective Hamiltonian (5.12) shares common global properties with the spin Hamiltonian (3.1): the dimension of space (i.e. dimension of vectors $\mathbf{k}, \mathbf{R}$ ), dimension of the order parameter $(m)$ and its symmetry: the functional representation we obtained is symmetric under group of rotations in the $m$-dimensional space $O(m)$ : it depends only on $|\vec{\phi}|$. Let us now see what differences will appear in the functional representation of the non-ideal models.

\subsection{Cubic model}

It is intuitively clear that an effective Hamiltonian of the cubic model (3.2) will differ from that of the $m$-vector model (5.12) by the presence of terms with new, cubic symmetry. Indeed this is the case that one can easily check following the next steps: starting from the Hamiltonian (3.2) via the Stratonovich-Hubbard transformation one obtains the functional representation for the interaction part (which coincides with equation (5.7)). An additional term $\mathrm{e}^{-\beta V \sum_{i} S^{i}}$ can be represented in a form of the functional derivative $\mathrm{e}^{-\beta V \sum_{i} \partial^{4} / \partial\left(\phi^{i}\right)^{4}}$, resulting in the following expression for the partition function of the cubic model (c.f. equation (5.9)):

$$
\mathcal{Z} \sim \int(\mathrm{d} \vec{\phi}) \mathrm{e}^{\frac{-1}{2} \sum_{\mathbf{k}} \frac{1}{\beta \nu(k)}\left|\vec{\phi}_{\mathbf{k}}\right|^{2}} \mathrm{e}^{-\beta V \sum_{\mathbf{R}} \sum_{i} \partial^{4} / \partial\left(\phi_{\mathbf{R}}^{i}\right)^{4}} \mathrm{Sp}^{\sum_{\mathbf{R}} \vec{S}_{\mathbf{R}} \vec{\phi}_{\mathbf{R}}}
$$

Taking trace in equation (5.13) leads to the familiar expression (5.10). However, the derivative $\partial^{4} / \partial\left(\phi_{\mathbf{R}}^{i}\right)^{4}$ gives rise to the contributions of cubic symmetry, the lowest order contribution being proportional to $v_{0} \sum_{i}\left(\phi_{\mathbf{R}}^{i}\right)^{4}$. The resulting effective Hamiltonian reads:

$$
\mathcal{H}_{\text {eff }}=\int \mathrm{d}^{d} R\left\{\frac{1}{2}\left((\nabla \phi)^{2}+\mu_{0}^{2} \phi^{2}\right)+\frac{u_{0}}{4 !} \phi^{4}+\frac{v_{0}}{4 !} \sum_{i=1}^{m}\left(\phi^{i}\right)^{4}\right\},
$$

and contains two bare couplings $u_{0}$ and $v_{0}$, corresponding to the $\phi^{4}$ terms of different symmetries. Coupling $u_{0}$ is positive, whereas the sign of the coupling $v_{0}$ coincides with that of $V$ in equation (3.2). At $v_{0}=0$ one recovers an effective Hamiltonian of the $m$-vector model (5.12).

\subsection{Weakly diluted quenched $m$-vector model}

The peculiarities of the free energy calculation for the model (3.3) consist in averaging over quenched disorder. Indeed, for each configuration of empty and occupied sites in (3.3) one can write a corresponding configuration-dependent partition function $Z_{\text {conf }}$ :

$$
\mathcal{Z}_{\text {conf }}=\operatorname{Sp}_{\vec{S}} \mathrm{e}^{-\beta \mathcal{H}}
$$


where $\operatorname{Sp}_{\vec{S}}$ concerns spin degrees of freedom and is defined by (5.2). The free energy is obtained as the configurational average:

$$
\mathcal{F}=-\beta^{-1}\left\langle\ln \mathcal{Z}_{\text {conf }}\right\rangle_{\text {conf }}
$$

One of the ways to proceed is to make use of the replica trick [25], which allows us to avoid integration of the logarithm in (5.16) substituting it by a power function:

$$
\ln \mathcal{Z}=\lim _{n \rightarrow 0} \frac{\mathcal{Z}^{n}-1}{n}
$$

Then $Z_{\text {conf }}^{n}$ can be written as:

$$
\mathcal{Z}_{\text {conf }}^{n}=\operatorname{Spe}^{\frac{\beta}{2} \sum_{\mathbf{R}, \mathbf{R}^{\prime}} J\left(\left|\mathbf{R}-\mathbf{R}^{\prime}\right|\right) \sum_{\alpha=1}^{n} \vec{\sigma}_{\mathbf{R}}^{\alpha} \vec{\sigma}_{\mathbf{R}^{\prime}}^{\alpha}}
$$

with obvious notations $\operatorname{Sp}(\ldots)=\prod_{\alpha=1}^{n} \operatorname{Sp}_{\vec{S}^{\alpha}}(\ldots)$ and $\vec{\sigma}_{\mathbf{R}}^{\alpha} \equiv c_{\mathbf{R}} \vec{S}_{\mathbf{R}}^{\alpha}$. Upcoming calculations closely follow lines of the subsection 5.1. Introducing by the Stratonovich-Hubbard transformation field variables $\vec{\phi}_{\mathbf{R}}^{\alpha}$, conjugated to $\vec{\sigma}_{\mathbf{R}}^{\alpha}$ one is able to take the trace over spin subsystem and is left with the configuration-dependent partition function:

$$
\mathcal{Z}_{\text {conf }}^{n} \sim \int(\mathrm{d} \vec{\phi}) \mathrm{e}^{\frac{-1}{2} \sum_{\mathbf{k}} \frac{1}{\beta \nu(k)} \sum_{\alpha=1}^{n} \vec{\phi}_{\mathbf{k}}^{\alpha} \vec{\phi}_{-\mathbf{k}}^{\alpha}-\sum_{\mathbf{R}} \sum_{l=1}^{\infty} \frac{u_{2 l} l}{(2 l) !} \sum_{\alpha=1}^{n}\left|\vec{\phi}_{\mathbf{R}}^{\alpha}\right|^{2 l} c_{\mathbf{R}}} .
$$

Note that $c_{\mathbf{R}}$ appears in (5.19) in the first power because from $c_{\mathbf{R}}=\{0,1\}$ it follows that $\left(c_{\mathbf{R}}\right)^{l} \equiv$ $c_{\mathbf{R}}$. The last step is to perform configurational averaging of (5.19) with the distribution function (3.5). Let us represent the result of averaging in the exponential form

$$
\left\langle\mathrm{e}^{-\sum_{\mathbf{R}} \sum_{l=1}^{\infty} \frac{u_{2 l}}{(2 l) !} \sum_{\alpha=1}^{n}\left|\vec{\phi}_{\mathbf{R}}^{\alpha}\right|^{2 l} c_{\mathbf{R}}}\right\rangle_{\text {conf }}=\prod_{\mathbf{R}} \mathrm{e}^{\sum_{p \geqslant 1} \frac{(-1)^{p}}{p !} \kappa_{p}(c)\left(\sum_{l=1}^{\infty} \frac{u_{2 l}}{(2 l) !} \sum_{\alpha=1}^{n}\left|\vec{\phi}_{\mathbf{R}}^{\alpha}\right|^{2 l}\right)^{p}}
$$

where $\kappa_{p}(c)$ are cumulants of random variables $c_{\mathbf{R}}$ and can be easily calculated for the random variable distribution $(3.5)$ :

$$
\begin{aligned}
\kappa_{1} & =\sum_{c_{\mathbf{R}}=\{0,1\}} c_{\mathbf{R}} p\left(c_{\mathbf{R}}\right)=c \\
\kappa_{2} & =\sum_{c_{\mathbf{R}}=\{0,1\}}\left(c_{\mathbf{R}}\right)^{2} p\left(c_{\mathbf{R}}\right)-\left(\sum_{c_{\mathbf{R}}=\{0,1\}} c_{\mathbf{R}} p\left(c_{\mathbf{R}}\right)\right)^{2}=c(1-c), \ldots
\end{aligned}
$$

For the free energy one gets:

$$
\mathcal{F}=-\beta^{-1}\left\langle\ln Z_{\text {conf }}\right\rangle_{\text {conf }}=-\beta^{-1} \lim _{n \rightarrow 0}\left\{\frac{c_{1}}{n} \int(\mathrm{d} \vec{\phi})^{n} \mathrm{e}^{-F[\phi]}-1 / n\right\}
$$

where the ( $n$-dependent) coefficient $c_{1}$ can be recast explicitly following all the steps of calculations described above and the free energy functional $F[\phi]$ is given by:

$$
F[\phi]=\frac{1}{2} \sum_{\mathbf{k}} \sum_{\alpha=1}^{n} \frac{1}{\beta \nu(k)}\left|\vec{\phi}_{\mathbf{k}}^{\alpha}\right|^{2}-\sum_{p \geqslant 1} \frac{(-1)^{p}}{p !} \kappa_{p}(c) \sum_{\mathbf{R}}\left(\sum_{l=1}^{\infty} \frac{u_{2 l}}{(2 l) !} \sum_{\alpha=1}^{n}\left|\vec{\phi}_{\mathbf{R}}^{\alpha}\right|^{2 l}\right)^{p} .
$$

Again, as in the previous subsection, restricting ourselves to the $\phi^{4}$ terms, expanding the shortrange interaction potential, re-scaling the fields and passing to the continuous limit one gets for the free energy:

$$
\mathcal{F} \sim \int(\mathrm{d} \vec{\phi}) \mathrm{e}^{-\mathcal{H}_{\text {eff }}}
$$


now the proportionality sign also hides, besides the coefficient, the replica limit, as written explicitly in (5.22). The effective Hamiltonian reads:

$$
\mathcal{H}_{\mathrm{eff}}=\int \mathrm{d}^{d} R\left\{\frac{1}{2} \sum_{\alpha=1}^{n}\left[\left(\nabla \phi^{\alpha}\right)^{2}+\mu_{0}^{2}\left(\phi^{\alpha}\right)^{2}\right]+\frac{u_{0}}{4 !} \sum_{\alpha=1}^{n}\left(\phi^{\alpha}\right)^{4}+\frac{v_{0}}{4 !} \sum_{\alpha, \beta=1}^{n}\left(\phi^{\alpha}\right)^{2}\left(\phi^{\beta}\right)^{2}\right\} .
$$

Here, the coupling $u_{0}$ is positive (being proportional to $c u_{4},(5.10)$ ) whereas the coupling $v_{0}$ is proportional to $c(c-1) u_{2}^{2}$ and is negative. The last term in (5.25) is present only for non-zero dilution: it is directly responsible for the effective interaction between replicas due to the presence of impurities.

\subsection{Random anisotropy model}

To treat the random anisotropy in the spin Hamiltonian (3.6), one first writes the configurationdependent partition function for a fixed local anisotropy axes configuration $\{\hat{x}\}$ :

$$
\mathcal{Z}_{\text {conf }}(\{\hat{x}\})=\operatorname{Sp}_{\vec{S}^{-\beta \mathcal{H}}} .
$$

As in equation (5.15), trace in equation (5.26) concerns only the spin degrees of freedom. Applying the Stratonovich-Hubbard transformation to the interaction part of the Hamiltonian one presents (5.26) in the form of the functional integral and is able to take the trace:

$$
\mathcal{Z}_{\text {conf }}(\{\hat{x}\}) \sim \int(\mathrm{d} \vec{\phi}) \mathrm{e}^{-\mathcal{H}(\hat{x})},
$$

with

$$
\mathcal{H}(\hat{x})=\int \mathrm{d}^{d} R\left\{\frac{1}{2}\left[(\nabla \phi)^{2}+\mu_{1}^{2} \phi^{2}\right]-D_{1}(\phi \hat{x})^{2}+v_{1} \phi^{4}+z_{1} \phi^{2}(\phi \hat{x})^{2}+\cdots\right\} .
$$

Again, the expansion for small $k$ was performed and the continuous limit has been taken. Explicit values for the coefficients $\mu_{1}, D_{1}, v_{1}, z_{1}$ are given in [20].

The rest of calculations follow the steps outlined in the section 5.3: for the quenched disorder the free energy is defined by (5.16), where averaging is performed over the random axis distribution (given by (3.7) or (3.8)). Subsequently, one substitutes $\ln \mathcal{Z}$ by a power function via the replica trick (5.17). For the isotropic random axis distribution (3.7) one gets the effective Hamiltonian [21] containing three $\phi^{4}$ couplings of different symmetry:

$$
\mathcal{H}_{\text {eff }}=\int \mathrm{d}^{d} R\left\{\frac{1}{2}\left[(\nabla \varphi)^{2}+\mu_{0}^{2} \varphi^{2}\right]+u_{0} \varphi^{4}+v_{0} \sum_{\alpha=1}^{n}\left(\phi^{\alpha}\right)^{4}+w_{0} \sum_{\alpha, \beta=1}^{n} \sum_{i, j=1}^{m} \phi_{i}^{\alpha} \phi_{j}^{\alpha} \phi_{i}^{\beta} \phi_{j}^{\beta}\right\} .
$$

Here and below $\varphi^{2}=\sum_{\alpha=1}^{n}\left(\phi^{\alpha}\right)^{2}$. One can check the signs of the couplings: $u_{0} \sim \frac{D^{2}}{m^{2}(m+2)}>0$, $v_{0} \sim D>0, w_{0} \sim \frac{-D^{2}}{m(m+2)}<0$. Moreover, from the explicit expressions for the couplings $w_{0}$ and $u_{0}$ one gets for their ratio $w_{0} / u_{0}=-m$. The latter relation determines a region of physically allowed initial values in the $(u-v-w)$-space of couplings.

For the cubic distribution (3.8) the average over the random variables $\{\hat{x}\}$ leads to the effective Hamiltonian [21] with four couplings:

$$
\begin{aligned}
\mathcal{H}_{\text {eff }}= & \int \mathrm{d}^{d} R\left\{\frac{1}{2}\left[(\nabla \varphi)^{2}+\mu_{0}^{2} \varphi^{2}\right]+u_{0} \varphi^{4}+v_{0} \sum_{\alpha=1}^{n}\left(\phi^{\alpha}\right)^{4}\right. \\
& \left.+w_{0} \sum_{i=1}^{m} \sum_{\alpha, \beta=1}^{n}\left(\phi_{i}^{\alpha}\right)^{2}\left(\phi_{i}^{\beta}\right)^{2}+y_{0} \sum_{i=1}^{m} \sum_{\alpha=1}^{n}\left(\phi_{i}^{\alpha}\right)^{4}\right\} .
\end{aligned}
$$


The signs of the couplings are as follows: $u_{0} \sim D^{2} /\left(2 m^{2}\right)>0, v_{0} \sim D>0, w_{0} \sim-D^{2} /(2 m)<0$. The last term in (5.30) is of cubic symmetry. It has to be included since it is generated if one further applies the RG transformation. Therefore $y_{0}$ can be of either sign. The symmetries of $w_{0}$ terms in (5.29) and (5.30) differ. However the ratio $w_{0} / u_{0}=-m$ holds for the effective Hamiltonian (5.30) as well.

\subsection{Stacked triangular antiferromagnet}

It is an interaction potential (3.9) that makes a difference in a derivation of the effective Hamiltonian for the frustrated magnet and the regular one. One can find the details of the procedure to derive an effective Hamiltonian via the Stratonovich-Hubbard transformation in [22]. The main difference from the above described calculations for the $m$-vector magnet (subsection 5.1) arises when one proceeds with the Gaussian term in equation (5.10):

$$
\sum_{\mathbf{k}}\left(\frac{1}{\beta \nu(k)}-u_{2}\right)\left|\vec{\phi}_{\mathbf{k}}\right|^{2} \equiv \sum_{\mathbf{k}} d(k)\left|\vec{\phi}_{\mathbf{k}}\right|^{2}
$$

The sum over $\mathbf{k}$ in (5.31) spans the 1 st Brillouin zone. For the $m$-vector model, $d(k)$ has a minimum at $k=0$ and an expansion in $k$ around this minimum results in the effective Hamiltonian (5.12). Note, that one minimum in $d(k)$ leads to one field variable $\phi_{\mathbf{k}}$ in the effective Hamiltonian. For the frustrated model, the $d(k)$ has a maximum at $k=0$ and two independent minima at $k \neq 0$ in the 1st Brillouin zone. Subsequently, the zone can be rearranged into separate subzones and the field $\phi_{\mathbf{k}}$ over the original Brillouin zone can be decomposed into two fields, $\phi_{1, \mathbf{k}}$ and $\phi_{2, \mathbf{k}}$, with $\mathbf{k}$ confined to the new subzone. Now, the minima of $d(k)$ occur at the origin and pertain to the fields $\phi_{1}$ and $\phi_{2}$. The above decomposition results in the appearance of the terms of new symmetry in the effective Hamiltonian, which now reads:

$$
\mathcal{H}_{\text {eff }}=\int \mathrm{d}^{d} R\left\{\frac{1}{2}\left[\mu_{0}^{2}\left(\phi_{1}^{2}+\phi_{2}^{2}\right)+\left(\nabla \phi_{1}\right)^{2}+\left(\nabla \phi_{2}\right)^{2}\right]+\frac{u_{0}}{4 !}\left[\phi_{1}^{2}+\phi_{2}^{2}\right]^{2}+\frac{v_{0}}{4 !}\left[\left(\phi_{1} \cdot \phi_{2}\right)^{2}-\phi_{1}^{2} \phi_{2}^{2}\right]\right\}
$$

with a scalar product of $m$-component fields $\phi_{1} \cdot \phi_{2}=\sum_{i=1}^{m} \phi_{1}^{i} \phi_{2}^{i}$. The coupling $u_{0}$ in (5.32) is positive, whereas the sign of the coupling $v_{0}$ determines a type of spin ordering: a non-collinear order occurs for $v_{0}>0$. For $v_{0}<0$ the fields $\phi_{1}$ and $\phi_{2}$ tend to allign parallel or antiparallel, this corresponds to the sinusoidal ordering or the linearly-polarized spin-density wave.

\section{RG explanation of criticality in non-ideal systems}

Now with the effective Hamiltonians (section 5) and the RG method (section 4) at hand one can proceed further, applying the method in order to study the critical behaviour of the models. However, to be honest with the reader, he should be warned that the whole story is not that simple as shown in the section 4 . It is not only because the very models we are interested in are much more complicated as compared to the 1d Ising model: in what follows below we will be interested in the criticality of $3 \mathrm{~d}$ systems. The real space renormalization described in the section 4 works for the low-dimensional systems, whereas for realistic $3 \mathrm{~d}$ systems one rather applies the RG equations in the momentum space. Moreover, the very approach to RG as to the way of calculating the partition function of the system [3] has its alternative known as the field-theoretical RG [4]. The latter is a tool to cope with the divergencies of correlation functions (vertex functions). Here, the RG procedure consists in the controlled rearrangement of the perturbation theory expansions giving rise to the RG equations. However, the underlying notions of both procedures are the same: given the effective Hamiltonian one applies the RG transformation and studies the flow equations for the couplings of the Hamiltonian. If the flow equations possess the fixed point (FP), which is stable and reachable from the initial conditions, it corresponds to the critical point of the system. Scaling arises in the vicinity of this FP and the universal values of the exponents governing scaling of different physical quantities may be found. Having this preamble in mind, we can make a brief 
review of what results for the RG flows, FPs and exponents of the non-ideal models have been found so far and how they differ from those of the "ideal" $m$-vector model.

\section{1. $m$-vector magnet}

Put in a formal way, the question: does the $3 \mathrm{~d} m$-vector magnet exhibit a critical point (a Curie point for a ferromagnet or a Néel point for an antiferromagnet), transforms into the question: is there a stable and reachable FP in the flow equations (cf. (4.14)-(4.16)) for the couplings of its effective Hamiltonian? The flow equation for the coupling $u$ of the effective Hamiltonian (5.12) can be written in a general form of an ordinary first order differential equation:

$$
\frac{\mathrm{d} u}{\mathrm{~d} \ln \ell}=\beta(u),
$$

with the RG flow parameter $\ell$ and $\beta$-function $\beta(u)$. The parameter $\ell$ may serve to measure a distance to the critical point: $\ell \rightarrow 0$ corresponds to $T \rightarrow T_{\mathrm{c}}$, and a specific form of the $\beta$-function depends on a choice of the RG procedure. The data discussed in this chapter have been obtained within the field-theoretic RG [4] approach at $d=3$. Giving numbers, we shall refer to the source, where they were obtained. However we shall try to be not too specific, mentioning several review papers for the interested reader.

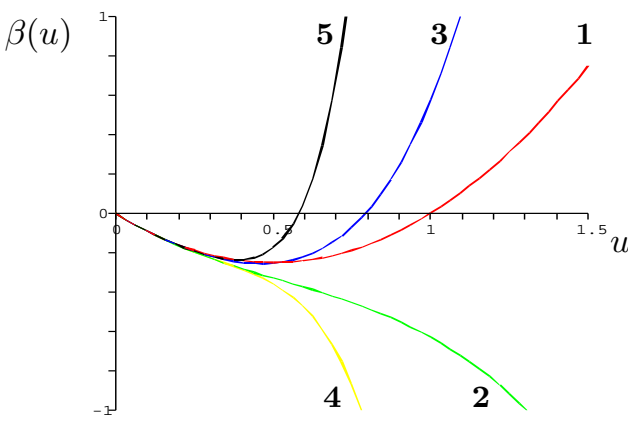

(a)

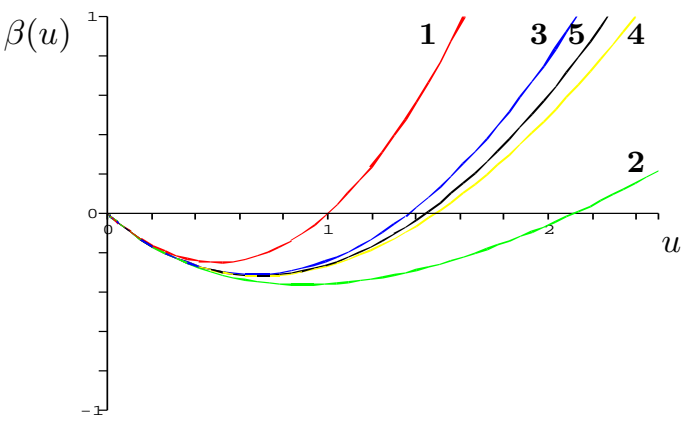

(b)

Figure 4. $\beta$-function of the $3 \mathrm{~d} m=1$ model in successive perturbation theory orders ranging from 1 to 5 as shown by the labels in the figures. Left: naive evaluation of the function (6.2). Right: resummation of (6.2) taking into account asymptotic properties of the series. Note a decrease of the difference between FP coordinates found in successive orders of the resummed perturbation expansion, figure $4 \mathbf{b}$.

To give an idea about the RG expansions and their treatment, we write down several first terms of the $\beta$-function (6.1) [26]:

$$
\beta(u)=-u\left(\varepsilon-u+3 u^{2}(3 m+14) /(m+8)+\cdots\right),
$$

with $\varepsilon=4-d$. For a quantitative analysis, one can develop an $\varepsilon$-expansion looking for solutions of the FP equation

$$
\beta\left(u^{*}\right)=0
$$

in a form of a series $u^{*}=\sum_{i} u^{(i)} \varepsilon^{i}$. Alternatively, one can solve the non-linear equation (6.3) directly at $d=3(\varepsilon=1)$. However, as it is well known by now, the perturbative RG expansions have zero radius of convergence and are asymptotic at best [4] (cf. behaviour of the function (6.2), figure $4 \mathbf{a}$ ). Special procedures of resummation have been elaborated to deal with them. We give an example of how one of these procedures works transforming a divergent series, figure $4 \mathbf{a}$, into 
a convergent one, figure $4 \mathbf{b}$. In the last figure, the function (6.2) has been resummed by the PadéBorel resummation. The procedure consists of several steps. First, assuming the factorial growth of the coefficients $c_{i}$ in the expansion $\beta(u)=\sum_{i} c_{i} u^{i}(6.2)$, one constructs the Borel trransform of the initial function $\beta(u)$ via:

$$
\beta^{\mathrm{B}}(u)=\sum_{i} \frac{c_{i} u^{i}}{i !} .
$$

Then, the Borel transform is extrapolated by a Padé approximant $[K / L](u)$. The last is the ratio of two polynomials of order $K$ and $L$ such that its truncated Taylor expansion is equal to $\beta^{\mathrm{B}}(u)$. The resummed function is then calculated by an inverse Borel transform of this approximant:

$$
\beta(u)=\int_{0}^{\infty} \mathrm{d} t \exp (-t)[K / L](u t)
$$

Similar techniques are currently widely used in analyzing the RG expansions [4,27]. In particular, the numerical estimates of different physical quantities given in this chapter (and in Table 1 as well) have been obtained using resummation techniques.

One sees the presence of two FPs in figure $4 \mathbf{b}$ : an unstable Gaussian FP $\mathbf{G} u^{*}=0$ (the slope of the $\beta$-function is negative, $\left.\partial \beta(u) /\left.\partial u\right|_{u^{*}}<0\right)$ and a stable one, $u^{*} \neq 0$. Here, the slope of the $\beta$-function is positive: starting form any initial conditions with $u>0$ the solution of the differential equation (6.1) reaches its FP value. This FP corresponds to the critical point $T_{\mathrm{c}}$ of the $3 \mathrm{~d}$ Ising model. Similar behaviour of the $\beta$-function is found for other values of $m$, therefore the FP with $u \neq 0$ for general $m$ is called a $O(m)$-symmetric or Heisenberg FP $\mathbf{H}$. We do not show the procedure of calculating the critical exponents: the other RG functions being evaluated at this FP bring about the asymptotic values of the critical exponents (in particular, those given in Table 1). These exponents govern criticality of systems of different microscopic nature, which can be described by the effective Hamiltonian (5.12). It is said that these systems belong to the $O(m)$ universality class. In the RG picture, different microscopic origin is reflected in different initial conditions for the flow. However, the FP location and stability is defined solely by the global features: dimensionality, symmetry, interaction type. ${ }^{3}$ Systems which share the global features belong to the same universality class.

\subsection{Cubic model}

The main difference of the effective Hamiltonian of the cubic model (5.14) as compared to that of the $m$-vector model (5.12) is that it contains one more coupling $v$ of different symmetry. Therefore, two $\beta$-functions describe the RG flow:

$$
\frac{\mathrm{d} u}{\mathrm{~d} \ln \ell}=\beta_{u}(u, v), \quad \frac{\mathrm{d} v}{\mathrm{~d} \ln \ell}=\beta_{v}(u, v) .
$$

Stability of a FP is now defined by the stability matrix composed of the $\beta$-function derivatives $\partial \beta_{u, v} / \partial\{u, v\}$. The FP picture, which arises form an analysis of the $\beta$-functions at $d=3$ [10] is schematically shown in figure 5. Four FPs are obtained: unstable Gaussian $\mathbf{G}(u=v=0)$ and Ising $\mathbf{I}(u=0, v \neq 0)$ as well as Heisenberg $\mathbf{H}(u \neq 0, v=0)$ and mixed $\mathbf{M}(u \neq 0, v \neq 0)$. The stability of the two last FPs depends on the value of $m$.

Two different regimes for the RG flows are observed. For small $m<m_{\mathrm{c}}^{\text {cub }}$ the FP $\mathbf{H}$ is stable. At this FP the system does not feel a presence of the cubic coupling, $v=0$. Therefore, for $m<m_{\mathrm{c}}^{\text {cub }}$ the cubic model belongs to the $O(m)$ universality class: its exponents coincide with the exponents of the $m$-vector model, table 1 . However, with an increase of $m$ the FP $\mathbf{M}$ approaches $\mathbf{H}$ and at $m=m_{\mathrm{c}}^{\text {cub }}$ both FPs coincide: a crossover to the new regime occurs. For $m>m_{\mathrm{c}}^{\text {cub }}$ the FP $\mathbf{M}$ becomes stable and governs the critical properties of the cubic model in the new universality class. The marginal dimension value is slightly less than three: $m_{\mathrm{c}}^{\mathrm{cub}}=2.862(5)$ [28]. From this estimate

\footnotetext{
${ }^{3}$ In particular, here we consider systems with the short-range interaction.
} 


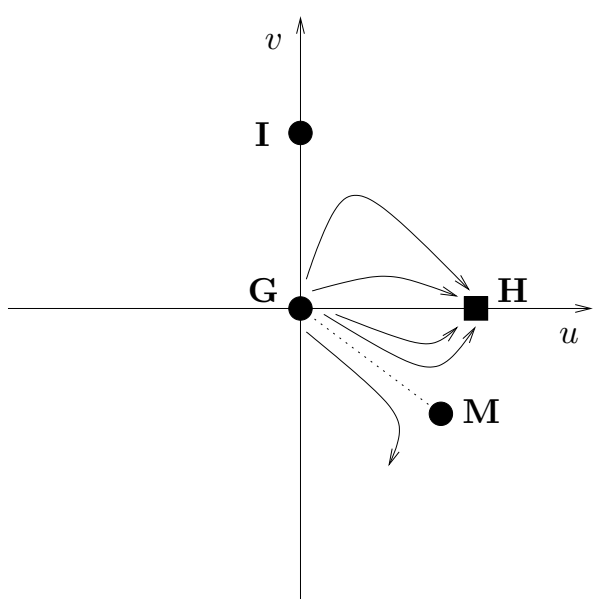

(a)

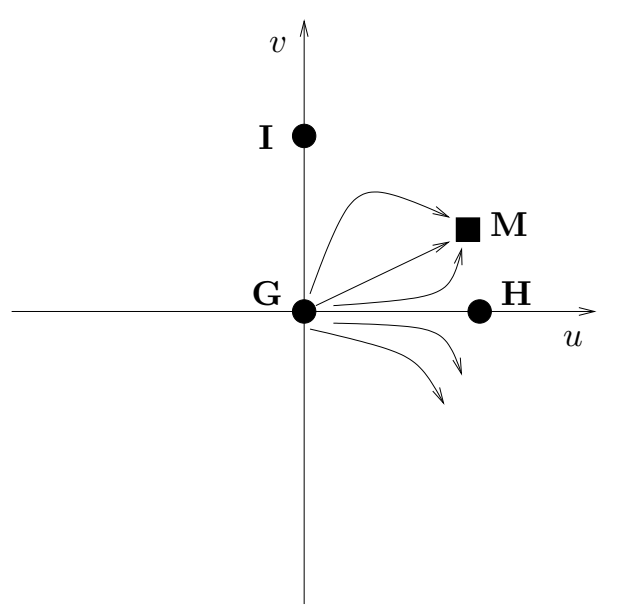

(b)

Figure 5. FPs and RG flows of the cubic model. Unstable FPs are shown by discs, stable FPs are shown by squares. (a): $m<m_{\mathrm{c}}^{\text {cub }}$, the Heisenberg FP $\mathbf{H}$ is stable. (b): $m>m_{\mathrm{c}}^{\text {cub }}$, the mixed FP $\mathbf{M}$ is stable. The dotted line in figure $5 \mathbf{a}$ shows a separatrix: the RG flows which start below this line do not reach $\mathbf{H}$. Note that for $m>m_{\mathrm{c}}^{\text {cub }}$ the $u$-axis is a separatrix: all flows that start from the initial conditions with $v<0$ do not reach any FP (run-away solutions).

it follows in particular, that a cubic Heisenberg $(m=3)$ magnet does not belong to the $O(3)$ universality class. Its critical exponent being estimated in the fixed point $\mathbf{M}$ read [29]:

$$
\gamma=1.390(12), \quad \nu=0.706(6), \quad \eta=0.0333(26), \quad \beta=0.364(15), \quad \alpha=-0.118(18) .
$$

Numerically, these values are close to their counterparts for the $m$-vector model (cf. Table 1). However the principal difference arises from the above analysis: as one can easily check solving the system of differential equations (6.6) for $m>m_{\mathrm{c}}^{\text {cub }}$ the RG flows with $v<0$ cannot reach the stable FP (an abscissa $v=0$ serves as a separatrix for the flows). As it follows from sections 3.2, 5.2 negative $v$ corresponds to ordering along diagonals of $m$-dimensional hypercube. Therefore, the RG analysis results in a statement that such ordering cannot occur via a 2nd order phase transition: ferromagnetic crystals with three easy axes should undergo a 1st order phase transition. It is worth noting here, that whereas the presence of stable and reachable FP brings about the 2nd order phase transition, its absence signalling only that the 2 nd order phase transition does not occur. The nature of the low-temperature phase and the scenario of how it is attained remains to be checked by other methods.

\subsection{Random-site dilution}

Formally, an analysis of the weakly diluted quenched $m$-vector model resembles those we discussed in the former subsection 6.2. Indeed, both effective Hamiltonians (5.14), (5.25) contain two couplings of different symmetry and give rise to the already familiar FP picture. Moreover, the effective Hamiltonian (5.25) at $m=1$ coincides with (5.14). ${ }^{4}$ However, our goal is to analyze it in the replica limit $n=0$. Note, that now the physically meaningful values of couplings are $u>0$, $v<0$, see section 5.3. The FP picture and the RG flows at $d=3$ are shown in the figure 6 [13]. Besides the familiar FPs $\mathbf{G}, \mathbf{H}$, and $\mathbf{M}$ a polymer FP $\mathbf{P}$ is present. It is stable and corresponds to the $O(m=0)$ universality class, however it is never reached from the initial conditions $u>0$, $v<0$.

Again, the new marginal dimension $m_{\mathrm{c}}^{\text {dil }}$ governs the crossover between the new and the $O(m)$ universality classes: FP $\mathbf{M}$ is stable for $m<m_{\mathrm{c}}^{\text {dil }}$. A search for the value of $m_{\mathrm{c}}^{\text {dil }}$ shows, that at $m=m_{\mathrm{c}}^{\text {dil }}$ the heat capacity critical exponent of an undiluted system changes its sign: $\alpha\left(m_{\mathrm{c}}^{\text {dil }}\right)=0$. In this way one recovers the Harris criterion [17], section 3.3.1, translated into the RG "language".

\footnotetext{
${ }^{4}$ One can check it by further substitution $n \rightarrow m,\left\{u_{0}, v_{0}\right\} \rightarrow\left\{v_{0}, u_{0}\right\}$.
} 


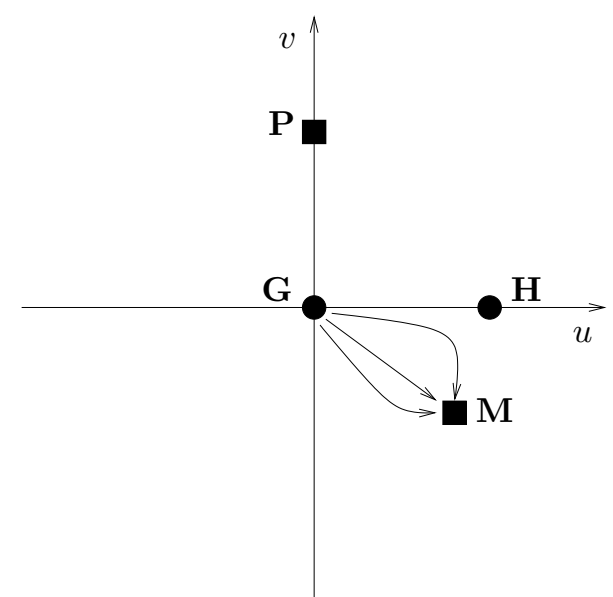

(a)

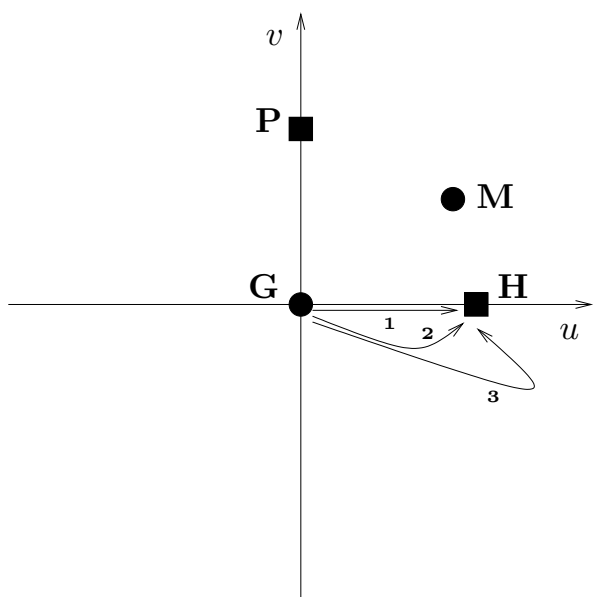

(b)

Figure 6. FPs and RG flows of the weakly diluted quenched $m$-vector model. Unstable FPs are shown by discs, stable FPs are shown by squares. (a): $m<m_{\mathrm{c}}^{\text {dil }}$, the mixed FP M is stable. (b): $m>m_{\mathrm{c}}^{\mathrm{dil}}$, the Heisenberg FP $\mathbf{H}$ is stable. Flows $1,2,3$ of the figure $6 \mathbf{b}$ are further treated in figure $7 \mathbf{b}$.

The numerical value of $m_{\mathrm{c}}^{\text {dil }}$ being slightly less than two, $m_{\mathrm{c}}^{\text {dil }}=1.912(4)$ [30], only the Ising model $(m=1)$ changes its exponents upon dilution. Indeed, the numerical values of the exponents $\operatorname{read}[31]$ :

$$
\gamma=1.330(17), \quad \nu=0.678(10), \quad \eta=0.030(3), \quad \beta=0.349(5), \quad \alpha=-0.034(30)
$$

and differ essentially from those of the $3 \mathrm{~d}$ Ising model (table 1 ).

However, both in the experiments and in the MC simulations one deals with the system not yet in an asymptotic region, where exponents do not attain their FP values and the effective exponents are found. Being non-universal, they can be calculated in the RG treatment as functions of the flow-dependent couplings. In figure 7 , we show an effective critical exponent $\gamma_{\text {eff }}$ measured recently for the ac susceptibility of the a-Fe ${ }_{86} \mathrm{Mn}_{4} \mathrm{Zr}_{10}$ amorphous alloy [32] and compare them with the theoretical RG calculations of the effective exponents of the weakly diluted quenched $m=3$ model. The exponent was calculated along different RG flows labeled by numbers in figure $6 \mathbf{b}$. This calculation serves an example how the non-asymptotic effects may be taken into account in the RG analysis. Although direct correspondence between the temperature distance to the critical point $\tau$ and the RG flow parameter $\ell$ is problematic, the RG serves as a useful tool of accompanying the studies of effective critical behaviour.

\subsection{Random anisotropy}

On this example we shall show how the RG predicts two different phenomena occuring in the random anisotropy magnets. It appears that the type of local random axis distribution crucially effects an origin of the low-temperature phase in random anisotropy systems [19]. First, we consider the results obtained for an isotropic distribution (3.7), which leads to the effective Hamiltonian (5.29) [21]. It contains three couplings, $u, v, w$, hence three $\beta$-functions define the RG flows. Solving the FP equations one arrives at the FP picture shown in figure $8 \mathbf{a}[21,20]$.

Let us recall (cf. section 5.4), that physically meaningful values of couplings are $u>0$, $v>0, w<0$. Therefore only the FPs located in this region are shown in the figure. However, there is another condition found for the ratio of couplings: $w_{0} / u_{0}=-m$. The region of typical initial conditions to study the RG flow is shown in figure 8 by a cross. The only stable FP found, a polymer FP $\mathbf{P}$ is not reachable from the initial conditions. The run-away solutions of the RG equations bring about an absence of a 2nd order phase transition.

A different picture is obtained for a cubic random axis distribution (3.8) [20]. Here, the effective Hamiltonian (5.30) contains four couplings of different symmetry, $u, v, w, y$. The physical initial 


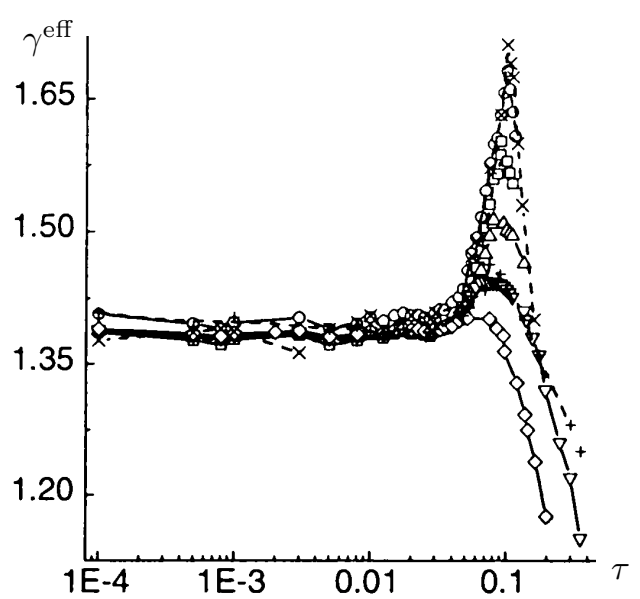

(a)

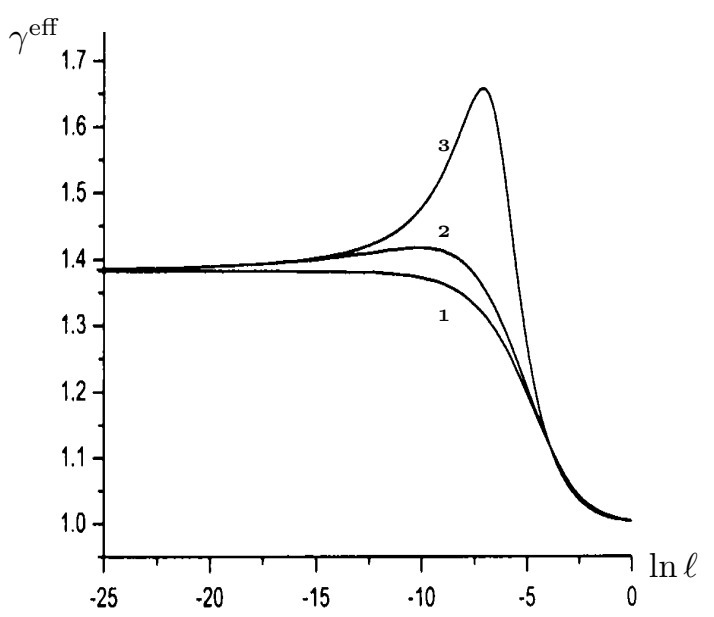

(b)

Figure 7. Effective critical exponent $\gamma^{\text {eff }}$ for the magnetic susceptibility of the weakly diluted quenched Heisenberg magnet. (a): as a function of the distance to the Curie point $\tau=\left(T-T_{\mathrm{c}}\right) / T_{\mathrm{c}}$ in experimental measurements for a-Fe $86 \mathrm{Mn}_{4} \mathrm{Zr}_{10}$ amorphous alloy [32]. (b): as a function of the RG flow parameter in theoretical RG calculations for the diluted $m=3$ model [15]. Different curves correspond to different amount of disorder. Note that in the asymptotics $(\tau \rightarrow 0$ or $\ell \rightarrow 0)$ the exponent attains its universal value.

values for the couplings lay in the region (section 5.4): $u>0, v>0, w<0, w / u=-m$. A typical FP picture is shown in figure $8 \mathbf{b}$ for $v=0$. Similar to the former case of isotropic random axis distribution, the stable FP $\mathbf{P}$ cannot be reached. However, one more stable FP $\mathbf{M}$ is present. It is reachable for the RG flow that starts from the initial conditions marked by a cross in the figure. This FP is a FP of the random-site Ising model (subsection 6.3) for any value of $m$. It means that the ferromagnetic 2 nd order phase transition in the $m$-vector magnet with the cubic random axis distribution belongs to the universality class of the random-site Ising model and is governed in asymptotics by the exponents (6.8).

\subsection{Stacked triangular antiferromagnet}

Again, as in the former subsections 6.1-6.4, the RG answer about a possibility of a 2nd order phase transition in the $3 \mathrm{~d}$ stacked triangular antiferromagnet would be a presence of a stable accessible FP for the couplings $u, v$ of the effective Hamiltonian (5.32). The model possesses a rather complicated FP structure, sketched in figure 9.

FP picture changes with $m$ and one finds three marginal dimensions $m_{i}^{\text {chir }}$ that govern its topology. For large $m>m_{3}^{\text {chir }}$ the stable chiral $\mathrm{FP} \mathbf{C}_{+}$is present and it can be reached from the initial values of the couplings $u, v \geqslant 0$. Therefore, the phase transition to the non-collinear chiral state is of the second order. At $m=m_{3}^{\text {chir }}, \mathbf{C}_{+}$merges with the anti-chiral FP $\mathbf{C}_{-}$and disappears, only one unstable Heisenberg FP $\mathbf{H}$ is found for $m_{3}^{\text {chir }}>m>m_{2}^{\text {chir }}$. With further decrease of $m$, two more FPs appear at $m=m_{2}^{\text {chir }}, \mathbf{S}_{-}$and $\mathbf{S}_{+}$. The last one is stable, however it describes the sinusoidal phase, which occurs for $v<0$. Finally, at $m=m_{1}^{\text {chir }}$ this FP merges with $\mathbf{H}$ and looses its stability with further increase of $m$. It follows from this analysis, that the 2 nd order phase transition into non-collinear phase can occur only in the magnets with $m>m_{3}^{\text {chir }}$.

In principle, the above described FP picture has been known since the first RG studies of the problem [22]. However, reliable numerical estimates for marginal dimensions $m_{i}^{\text {chir }}$ have been obtained only recently. The question of interest is, what is the order of the phase transition at $m=$ $2 ; 3$ when the model has its physical realization? The estimates: $m_{3}^{\text {chir }}=6.23(21), m_{2}^{\text {chir }}=1.99(4)$, $m_{1}^{\text {chir }}=1.43(2)$ [33] clearly rule out the possibility of a 2 nd order phase transition for $m=2 ; 3$ : the FP picture is shown in the second figure of figure 9 and no accessible FP is found.

Note, however, certain controversy in the RG studies performed so far: whereas the above FP picture is confirmed by the perturbative RG expansions and the non-perturbative RG approach 


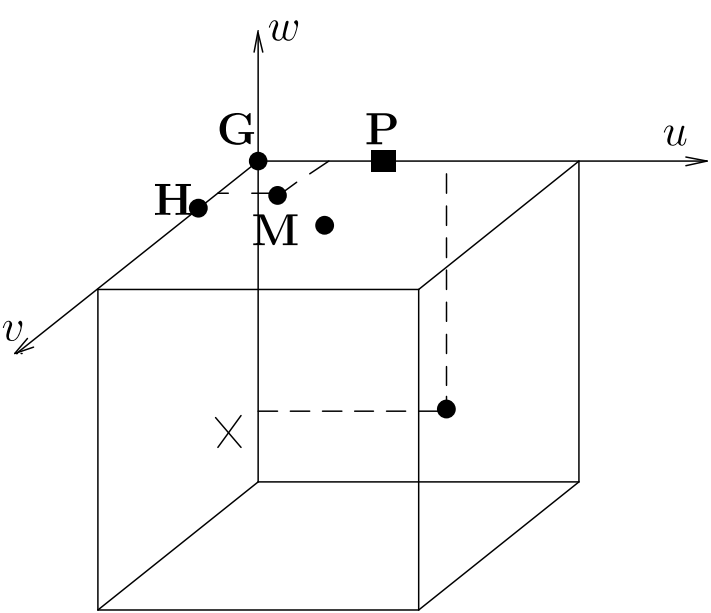

(a)

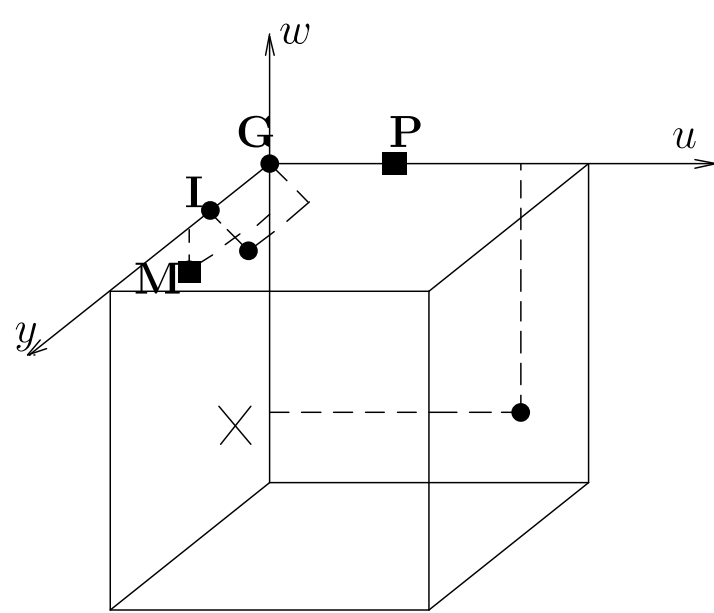

(b)

Figure 8. Fixed points of the random anisotropy model. The filled boxes show the stable fixed points, the cross denotes typical initial values of couplings. (a): isotropic local random axis distribution. The stable FP $\mathbf{P}$ cannot be reached by the RG flow which starts from the region shown by a cross in the figure. (b): cubic local random axis distribution. The stable FP $\mathbf{P}$ cannot be reached, but the random Ising FP M is stable and reachable for the RG flow.

[23,33], an analysis of the FP equations by direct solution of the non-linear FP equations for the resummed $\beta$-functions brings about a presence of the stable reachable FP for $m=2 ; 3$ [34]. The last is associated with the critical point of the 2 nd order phase transition.

\section{Conclusions and outlook}

How do the changes in structure effect the critical behaviour of the matter? We tried to give an answer to this question taking a $3 \mathrm{~d} m$-vector model as an ideal reference system and showing what will happen to it under an effect of different non-idealities. The examples considered include anisotropy, structural disorder, frustrations: the features one often encounters dealing with realistic condensed matter objects. The response of a system to such non-idealities appears to be very different, ranging from an insensitivity (the cubic $m=2$ magnet remains in the $O(2)$ universality class), through softening (the heat capacity of the random site $m=1$ magnet does not diverge) to disappearance of a 2 nd order phase transition (isotropically distributed local random axis destroys long-range order). These various asymptotic features are accompanied by a complicated non-asymptotic effective critical behaviour.

It is astonishing that all this bunch of phenomena can be explained and accurately described within one theoretical framework, the RG approach. An application of basic RG notions of flows, fixed points and their accessibility, marginal dimensions and crossovers supported by an elaborate machinery to perform and analyze the RG transformation resulted in a coherent picture of phenomena in the vicinity of a critical point. A lot remains to be cleared up in this picture. Maybe a participant of the school or a reader of these lectures will decide to make his or her contribution? Good luck!

\section{Acknowledgements}

I am grateful to Bertrand Berche, Arnaldo Donoso, and Ricardo Paredes for the invitation to lecture at the Spring school on Foundations of statistical and mesoscopic physics (Mochima, Venezuela, June 20th-24th 2005) and to all participants of the school for the wonderful atmosphere created there. Bertrand Berche is further acknowledged for his encouragement, advice during preparation of the manuscript and, last but not least, for his stories about the Cagniard de la Tour 

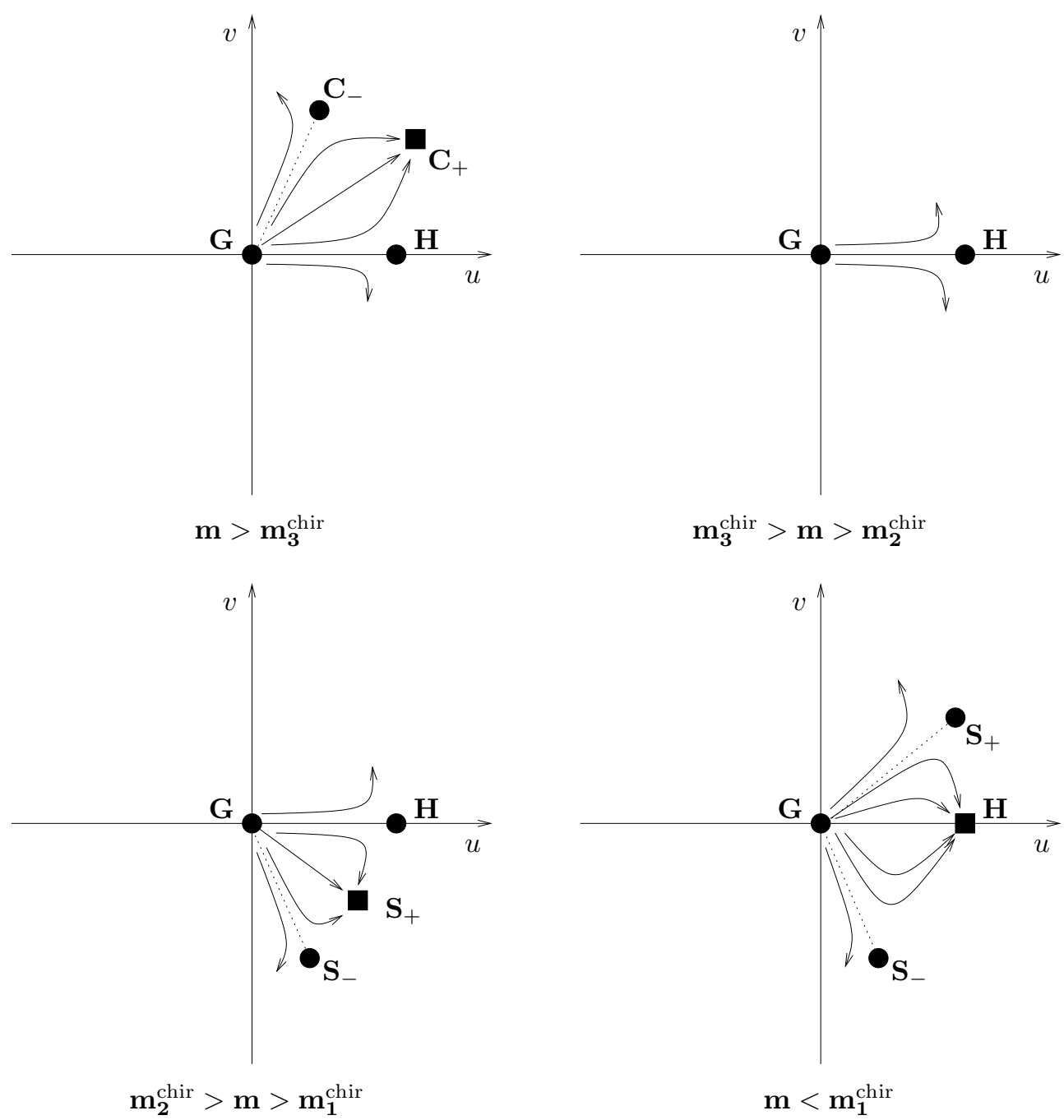

Figure 9. FPs and RG flows of the stacked triangular antiferromagnet model. Unstable FPs are shown by discs, stable FPs are shown by squares. Three marginal dimensions $m_{1}^{\text {chir }}, m_{2}^{\text {chir }}, m_{3}^{\text {chir }}$ govern the FP picture.

state, the critical state discovered as early as in 1822 [1]! I thank my colleagues Viktoria Blavats'ka, Bertrand Delamotte, Maxym Dudka, Christian von Ferber, Reinhard Folk, Dmytro Ivaneiko, Taras Yavors'kii - some of the results mentioned in the last part of these lectures are due to our common work.

This work was supported by Austrian Fonds zur Förderung der wissenschaftlichen Forschung under Project No. P16574.

\section{References}

1. A historical overview of the experimental and theoretical studies of critical phenomena may be found in J.S. Rowlinson introductary essay to the English edition of J.D. van der Waals Leiden thesis of 1873: J.D. van der Waals: On the Continuity of the Gaseous and Liquid States. Edited with an Introductory Essay by J.S. Rowlinson. North-Holland Physics Publishing, Amsterdam, 1988; and in: C. Domb. The Critical Point. Taylor \& Francis, London, 1996.

2. J.E. Verschaffelt. Koninklijke Akademie van Wetenschappen, Proc. Sect. Scienc., 1990, II, 588. English version: http://www.knaw.nl/waals/pdf/Verschaffelt01.pdf 
3. Wilson K.G., Kogut J., Phys. Rep., 1974, 12, 75.

4. The field theoretical RG in its applications to the phase transition theory is explained in: E. Brézin, J. C. Le Guillou, and J. Zinn-Justin, in: Phase Transitions and Critical Phenomena, edited by C. Domb and M. S. Green, Vol. 6, Academic Press, London, 1976; Amit D.J. Field Theory, the Renormalization Group, and Critical Phenomena. World Scientific, Singapore, 1989; Zinn-Justin J. Quantum Field Theory and Critical Phenomena. Oxford University Press, Oxford, 1996.

5. Ising E., Z. Phys., 1925, 31, 255.

6. Stanley H.E., Phys. Rev. Lett., 1968, 20, 589.

7. Mermin N.D., Wagner H., Phys. Rev. Lett., 1966, 17, 1133; Erratum: ibid., 1966, 17, 1307; Hohenberg P.C., Phys. Rev., 1967, 158, 383.

8. Onsager L., Phys. Rev., 1944, 65, 117.

9. Guida R., Zinn-Justin J., J. Phys. A, 1998, 31, 8103.

10. Aharony A. Phase Transitions and Critical Phenomena, edited by C. Domb and M. S. Green, Vol.6. Academic Press, London, 1976.

11. Brout R., Phys. Rev., 1959, 115, 824.

12. Belanger D.P., Young A.P., J. Magn. Magn. Mater., 1991, 100, 272.

13. A review of the random $3 \mathrm{~d}$ Ising model critical behaviour may be found in: Folk R., Holovatch Yu., Yavors'kii T., Physics - Uspekhi, 2003, 46, 169 [Uspekhi Fizicheskikh Nauk, 173, 175] and in [14].

14. A review on the critical behaviour of systems with complex effective Hamiltonians: Pelissetto A., Vicari E., Phys. Rep., 2002, 368, 549.

15. Dudka M., Folk R., Holovatch Yu., Ivaneiko D., J. Magn. Magn. Mater., 2003, 256, 243.

16. Yoon J., Chan M.H.W., Phys. Rev. Lett., 1997, 78, 4801.

17. Harris A.B., J. Phys. C, 1974, 7, 1671.

18. Harris R., Plischke M., Zuckermann M.J., Phys. Rev. Lett., 1973, 31, 160.

19. A review of early work on random anisotropy magnets may be found in: Cochrane R.W., Harris R., Zuckermann M.J., Phys. Rep., 1978, 48, 1. Recent experimental, numerical, and theoretical studies are reviewed in $[20]$.

20. Dudka M., Folk R., Holovatch Yu., Journ. Mag. Mag. Mat., 2005, 294, 305.

21. Aharony A., Phys. Rev. B, 1975, 121038.

22. Kawamura H., Phys. Rev. B, 1988, 384916.

23. A review on frustrated magnets with an emphasis on the results obtained within the non-perturbative RG approach: Delamotte B., Mouhanna D., Tissier M., Phys. Rev. B, 2004, 69, 134413.

24. Fisher M.E., in: Critical Phenomena. Proceedings of the Summer School, Stellenbosch, South Africa. Ed. by F. J. W. Hahne. Lecture Notes in Physics, 186, Springer Verlag, Berlin, 1983; D. R. Nelson, Fisher M.E., Ann. Phys. (N.Y.), 1975, 91, 226.

25. Emery V.J., Phys. Rev. B, 1975, 11, 239; Edwards S.F., Anderson P.W., J. Phys. F, 1975, 5, 965.

26. Kleinert H., Neu J., Schulte-Frohlinde V., Chetyrkin K.G., Larin S.A., Phys. Lett. B, 1991, 272, 39; Erratum: ibid., 1993, 319545.

27. A review on application of resummation technique to divergent RG expansions of disordered models: Holovatch Yu., Blavats'ka V., Dudka M., von Ferber C., Folk R., Yavors'kii T., Int. J. Mod. Phys. B, 2002, 16, 4027.

28. Folk R., Holovatch Yu., Yavors'kii T., Phys. Rev. B, 2000, 62, 12195.

29. Carmona J.M., Pelissetto A., Vicari E., Phys. Rev. B, 2000, 61, 15136.

30. Holovatch Yu., Dudka M., Yavors'kii T., J. Phys. Stud., 2001, 5, 233.

31. Pelissetto A., Vicari E., Phys. Rev. B, 2000, 62, 6393.

32. Perumal A., Srivinas V., Rao V.V., Dunlap R.A., Phys. Rev. Lett., 2003, 91, 137202.

33. Holovatch Yu., Ivaneiko D., Delamotte B., J. Phys. A, 2004, 37, 3569.

34. Pelissetto A., Rossi P., Vicari E., Phys. Rev. B, 2001, 63, 140414.

\section{Questions and answers}

$\mathcal{Q}$ (Alexander López): How do the signs of the couplings $u$ and $v$ in the effective Hamiltonian (5.14) determine a type of the low-temperature ordering?

$\mathcal{A}$ Neglecting fluctuations (taking a function $\phi(r)$ to be just a variable $\phi$ ) you can think about the effective Hamiltonian as of the Landau free energy. Now, let the reference system display a 2nd order phase transition. This means that $u>0$. Minimizing Landau free energy and looking for the spontaneous magnetization one finds at $T<T_{\mathrm{c}}$ two different non-trivial 
solutions: $\vec{\phi}=(\phi / \sqrt{m}, \ldots, \phi / \sqrt{m})$, it exists at $v<0$, and $\vec{\phi}=(\phi, 0, \ldots, 0)$ at $v>0$. They correspond to two types of ordering: along the diagonals or along the edges of a $m$-dimensional hypercube. For $m=3$, these are directions [111] and [100] correspondingly.

$\mathcal{Q}$ (Bertrand Berche): What are experimental realizations of the cubic model?

$\mathcal{A}$ I have already mentioned ferromagnetic crystals. Besides, at $m=3$ the model describes a ferroelectric phase transition which occurs in $\mathrm{SrTiO}_{3}$ at $105 \mathrm{~K}$ ( Cowley R.A., Bruce A.D., J. Phys. C, 1973, 6, L191). Moreover, since the model provides an example of a system with an arbitrary weak first-order phase transition, it is also used as a testing ground to describe an elecroweak transition in the early Universe (P. Arnold, S Sharpe, L. Yaffe, Y. Zhang, Phys. Rev. Lett., 1997, 78, 2062).

$\mathcal{Q}$ (Carlos Vásquez): In 1983, Weinrib and Halperin proposed a model to describe an effect of extended (correlated) disorder on magnetic 2nd order phase transition. There, the impurityimpurity correlation function decays for large separations as $g(r) \sim r^{-a}$. Currently, there exist two different predictions for the critical exponents of such a model. What are the methods used to obtain them?

$\mathcal{A}$ Indeed, the original result of Weinrib and Halperin ( A. Weinrib, Halperin B.I., Phys. Rev. B, $1983, \mathbf{2 7}, 417)$ was obtained in the first order of the expansion in $\varepsilon=4-d, \delta=4-a$. Recent two-loop estimates (V. V. Prudnikov, Prudnikov P.V., Fedorenko A.A., J. Phys. A, 1999, 32, L399) are due to the fixed $d, a$ RG technique. Qualitative answer of both approaches is that for $a<d$ the disorder is relevant if the correlation length critical exponent of the system without defects obeys $\nu<2 / a$.

$\mathcal{Q}$ (Bertrand Berche): Could you comment on logarithmic corrections to the scaling laws? Is the critical behaviour at marginal dimensions you were speaking about (e.g. at $m_{\mathrm{c}}^{\text {dil }} \simeq 1.91$ for the $3 \mathrm{~d}$ diluted $m$-vector magnet) governed by such corrections?

$\mathcal{A}$ Logarithmic corrections arise at upper critical dimension $d^{\text {up }}$ : that is, at the space dimension, above which the mean-field theory holds ${ }^{5}$. For the models I was speaking about, the upper critical dimension is four. Indeed, the divergence of an isothermal susceptibility of the $m$ vector model at $d=4$ is governed by the mean field exponent $\gamma=1$. However, the power law singularity is accompanied by a logarithmic one:

$$
\chi^{-1} \sim|\tau|^{-1} \ln |\tau|^{-(m+2) /(m+8)},
$$

as first derived in: Larkin A.I., Khmelnitskii D.E., JETP, 1969, 29, 1123. In the RG scheme, one can see the origin of such corrections solving the flow equation $(6.1)$ at $d=4(\varepsilon=0)$ :

$$
\frac{\mathrm{d} u}{\mathrm{~d} \ln \ell}=-u^{2},
$$

here the right-hand side is the leading term of the $\beta$-function (6.2). The solution of equation $(7.2)$

$$
u=\frac{1}{|\ln \ell|}+\text { const }, \quad \ell \rightarrow 0
$$

being substituted into an appropriate expression for the susceptibility leads to the above dependence (7.1). Now, let us return to the marginal dimensions $m_{\mathrm{c}}$ we were discussing in these lectures. They are field dimensions and we estimated them at space dimension $d=3$. As far as the space dimension was lower than $d^{\text {up }}=4$, the logarithmic corrections do not appear. Returning back to the example given by formulas (7.2), (7.3): even at $m=m_{\mathrm{c}}$, the first power of couplings is present in the right-hand side of functions (7.2) for $d=3$ and the solutions will rather behave as $u \sim \ell^{\text {const }}$.

${ }^{5}$ Note added in proof: We do not discuss here the logarithmic corrections appearing at low dimensions as those in $2 \mathrm{~d}$ diluted Ising model or $2 \mathrm{~d} q=4$ Potts model. For a list of systems where logarithmic corrections appear and for the scaling relations between them see: Kenna R., Johnston D.A., Janke W., Phys. Rev. Lett., 2006, 96, 115701. 
$\mathcal{Q}$ (Dragi Karevski): Do you know other citeria, similar to the Harris one, which predict changes in the critical behaviour caused by different types of disorder? For example, what happens when disorder is coupled to the order parameter?

$\mathcal{A}$ Indeed, Harris criterion concerns the systems, where disorder is coupled to the energy density (look for example at the Hamiltonian (3.3), where random variables $c_{\mathbf{R}}$ are coupled to the product of spins). It states that critical exponents of a disordered system do not change, if the heat capacity of the pure system does not diverge. Later, a statement that the correlation length critical exponent of $d$-dimensional systems with such type of disorder should obey an inequality $\nu>2 / d$ was proven ( Chayes J.T., L. Chayes, Fisher D.S., T. Spenser, Phys. Rev. Lett., 1986, 57, 2999). For the extended, long-range correlated disorder the generalized Harris criterion holds (see the above mentioned paper of Weinrib and Halperin as well as D. Boyanovsky, Cardy J.L., Phys. Rev. B, 1983, 27, 6971).

Y. Imry and S.-k Ma have shown (Phys. Rev. Lett., 1975, 35, 1399) that even arbitrary weak disorder coupled to the order parameter of continuous symmetry (i.e. for $m \geqslant 2$ vector model) destroys ferromagnetism at $d<4$. For the random-field Ising model $(m=1)$ the lower critical dimension is $d=2$. As I already have mentioned in the lectures, the isotropically distributed random axis destroys ferromagnetism at $d<4$. One of the ways of showing this is to exploit the arguments similar to those of Imry and Ma for the random-field systems (Pelcovits R.A., Pytte E., Rudnick J., Phys. Rev. Lett., 1978, 40, 476).

Last but not least, let me mention the Luck criterion for the connectivity disorder, as explained in the lecture by Wolfhard Janke.

\title{
Вступ до перенормування
}

\author{
Ю.Головач ${ }^{1,2,3}$ \\ 1 Інститут фізики конденсованих систем НАН України, 79011 Львів, вул. Свєнціцького, 1, Україна \\ 2 Інститут теоретичної фізики, Университет Йогана Кеплера міста Лінц, Лінц 4040, Австрія \\ 3 Львівський національний університет ім. І.Франка, Львів 79005, Україна
}

Отримано 13 лютого 2006 р., в остаточному вигляді - 17 квітня 2006 р.

В цих лекціях я обговорюю особливості критичної поведінки "неідеальних" систем і те, як їх можна пояснити за допомогою методу ренормалізаційної групи. Розглядаються системи з однорідною анізотропією, структурним безладом, фрустраціями. Я запроваджую основні ідеї перенормування і показую, як вони служать для пояснення типових рис критичності в таких системах: пом'якшення фазового переходу, зміни класу універсальності, складної ефективної критичної поведінки.

Ключові слова: критична поведінка, перенормування, теоретико-польова ренормалізаційна група

PACS: $05.50 .+q, 05.70 . J k, 64.60 . A k$ 